\title{
Metformin inhibits hepatic gluconeogenesis in mice independently of the LKB1/AMPK pathway via a decrease in hepatic energy state
}

\author{
Marc Foretz,1,2 Sophie Hébrard,,1,2 Jocelyne Leclerc,1,2 Elham Zarrinpashneh, ${ }^{3}$ Maud Soty, ${ }^{4,5,6}$ \\ Gilles Mithieux, ${ }^{4,5,6}$ Kei Sakamoto, ${ }^{3}$ Fabrizio Andreelli,1,2,7 and Benoit Viollet'1,2

\begin{abstract}
${ }^{1}$ Institut Cochin, Université Paris Descartes, CNRS (UMR 8104), Paris, France. 2INSERM, U1016, Paris, France. ${ }^{3}$ MRC Protein Phosphorylation Unit, College of Life Sciences, University of Dundee, Dundee, United Kingdom. ${ }^{4}$ INSERM, U855, Lyon, France. ${ }^{5}$ Université de Lyon, Lyon, France. ${ }^{6}$ Université Lyon I, Villeurbanne, France. ${ }^{7} \mathrm{CHU}$ Bichat Claude Bernard, Service de Diabétologie-Endocrinologie-Nutrition, AP-HP, Paris, France.
\end{abstract}

\begin{abstract}
Metformin is widely used to treat hyperglycemia in individuals with type 2 diabetes. Recently the LKB1/AMPactivated protein kinase (LKB1/AMPK) pathway was proposed to mediate the action of metformin on hepatic gluconeogenesis. However, the molecular mechanism by which this pathway operates had remained elusive. Surprisingly, here we have found that in mice lacking AMPK in the liver, blood glucose levels were comparable to those in wild-type mice, and the hypoglycemic effect of metformin was maintained. Hepatocytes lacking AMPK displayed normal glucose production and gluconeogenic gene expression compared with wild-type hepatocytes. In contrast, gluconeogenesis was upregulated in LKB1-deficient hepatocytes. Metformin decreased expression of the gene encoding the catalytic subunit of glucose-6-phosphatase (G6Pase), while cytosolic phosphoenolpyruvate carboxykinase (Pepck) gene expression was unaffected in wild-type, AMPK-deficient, and LKB1-deficient hepatocytes. Surprisingly, metformin-induced inhibition of glucose production was amplified in both AMPK- and LKB1-deficient compared with wild-type hepatocytes. This inhibition correlated in a dose-dependent manner with a reduction in intracellular ATP content, which is crucial for glucose production. Moreover, metformin-induced inhibition of glucose production was preserved under forced expression of gluconeogenic genes through PPAR $\gamma$ coactivator $1 \alpha(\mathrm{PGC}-1 \alpha)$ overexpression, indicating that metformin suppresses gluconeogenesis via a transcription-independent process. In conclusion, we demonstrate that metformin inhibits hepatic gluconeogenesis in an LKB1- and AMPK-independent manner via a decrease in hepatic energy state.
\end{abstract}

\section{Introduction}

Type 2 diabetes is a progressive metabolic disorder with diverse pathological manifestations and is often associated with abnormal lipid and glucose metabolism. Metformin is currently the drug of first choice for the treatment of type 2 diabetes, being prescribed to at least 120 million people worldwide. As demonstrated in large prospective controlled clinical trials, metformin improves glycemic control and reduces cardiovascular mortality in overweight type 2 diabetic patients (1) and is also used to prevent type 2 diabetes (2). However, the molecular mechanisms of metformin action are not well understood. It was initially suggested that one of the key actions of metformin was to stimulate muscle glucose uptake (3). Recently, a growing body of evidence from clinical studies and animal models suggests that the primary function of metformin is to decrease hepatic glucose production, mainly by inhibiting gluconeogenesis (4-6). This preferential action of metformin in hepatocytes is due to the predominant expression of organic cation transporter 1 (OCT1), which has been shown to facilitate cellular uptake of metformin. Consistent with this notion, deletion of the Oct1 gene in mouse dramatically reduces metformin uptake in hepatocytes, and humans carrying reduced function polymorphisms of the OCT1 gene display an impaired effect of metformin in lowering blood glucose levels (7).

Conflict of interest: The authors have declared that no conflict of interest exists. Citation for this article: J Clin Invest. 2010;120(7):2355-2369. doi:10.1172/JCI40671.
Although the molecular target of metformin was elusive for several years, Zhou et al. demonstrated in 2001 that metformin treatment activates the energy sensor AMP-activated protein kinase (AMPK) in rat primary hepatocytes (8), and thereafter numerous groups also showed that metformin treatment stimulates AMPK in tissues in both humans and rodents $(9,10)$. AMPK is a phylogenetically conserved serine/threonine protein kinase composed of a catalytic $\alpha$-subunit and two regulatory subunits, $\beta$ and $\gamma$. In mammals, each AMPK subunit appears in multiple isoforms $(\alpha 1$, $\alpha 2, \beta 1, \beta 2, \gamma 1, \gamma 2$, and $\gamma 3$ ) that differ in tissue and subcellular localization, suggesting different roles (11). AMPK has been identified as a key regulator of cellular energy status and plays a crucial role in protecting cellular function under energy-restricted conditions in the liver (12). Thus, AMPK is activated in response to a variety of metabolic stresses that typically change the cellular AMP/ATP ratio caused by increasing ATP consumption or reducing ATP production, as seen following hypoxia, glucose deprivation, and inhibition of mitochondrial oxidative phosphorylation. AMPK activation requires phosphorylation on Thr172 within the activation loop of the catalytic $\alpha$-subunit by upstream kinases, identified as the tumor suppressor serine/threonine kinase 11 (STK11/LKB1) $(13-15)$ and CaMKK $\beta(16,17)$, which is further stimulated by the allosteric activator AMP (11). Activated AMPK switches cells from an anabolic to a catabolic state, shutting down the ATP-consuming synthetic pathways and restoring energy balance. 
The glucose-lowering effect of metformin has been mainly attributed to its ability to suppress hepatic gluconeogenesis through the signaling pathway downstream of LKB1 (10). The LKB1 pathway has been reported to regulate the phosphorylation and nuclear exclusion of CREB-regulated transcription coactivator 2 (CRTC2, also referred to as TORC2) (10). CRTC2 has been identified as a pivotal regulator of hepatic glucose output in response to fasting by directing transcriptional activation of the gluconeogenic program (18). Under feeding conditions, CRTC2 is sequestered in the cytoplasm; however, in response to fasting stimuli, CRTC2 is dephosphorylated and transported to the nucleus, where it enhances the transcriptional activation of the gluconeogenic genes. This transcriptional coactivator mediates CREB-dependent transcription of PPAR $\gamma$ coactivator- $1 \alpha(P g c-1 \alpha)$ and its subsequent gluconeogenic target genes, phosphoenolpyruvate carboxykinase (Pepck) and glucose-6-phosphatase (G6Pase). Phosphorylation on the Ser171 residue of CRTC2 by AMPK and AMPK-related kinases, including the salt-inducible kinases (SIKs), is critical for determining the activity, cellular localization, and degradation of CRTC2 (18-20). However, identification of a second regulatory phosphorylation site (Ser275) on CRTC2, mediated by the AMPK-related protein kinase $\mathrm{MAP} /$ microtubule affinity-regulating kinase 2 (MARK2), suggests that multiple signaling pathways converge to control CRTC2 activity (21). In addition, recent findings indicate that the regulation of gluconeogenic gene expression by metformin is dependent on the phosphorylation of CREB-binding protein (CBP), but not CRTC2, through atypical PKCı/ $\lambda$ (22), suggesting the complexity of the mechanism of metformin action.

Since metformin stimulates the activity of AMPK (8) via an LKB1-dependent mechanism, but not AMPK-related kinases in cells $(23,24)$, we hypothesized that the ability of metformin to suppress hepatic glucose production is mainly mediated via AMPK. To test this, we employed hepatocytes lacking either AMPK $\alpha 1 \alpha 2$ catalytic isoforms or LKB1. Here, we report that metformin inhibits hepatic glucose production through a mechanism linked to perturbation of intracellular ATP levels rather than direct inhibition of gluconeogenic gene expression. Furthermore, we provide genetic evidence that neither AMPK nor LKB1 is essential for metformin inhibition of hepatic glucose production.

\section{Results}

Metformin suppresses hepatic glucose production in the absence of AMPK catalytic subunits. Type 2 diabetic patients on metformin treatment $(20 \mathrm{mg} / \mathrm{kg})$ display plasma concentrations of metformin from $10 \mu \mathrm{M}$ to $40 \mu \mathrm{M}$ (25). However, it should be noted that the liver receives the majority of its blood via the portal vein, which may contain concentrations of metformin substantially higher than those present in the general circulation (26). In addition, due to the high expression of the cationic transporter OCT1 in the liver (7), metformin accumulates to significantly higher levels in hepatocytes than in plasma (27). Metformin liver concentration of greater than $180 \mu \mathrm{M}$ and $250 \mu \mathrm{M}$ in normal and diabetic rodents, respectively, can be achieved after a dose of $50 \mathrm{mg} / \mathrm{kg}$ (26). To study the effect of metformin on gluconeogenesis in primary culture of hepatocytes, we chose metformin concentrations between $250 \mu \mathrm{M}$ and $1 \mathrm{mM}$, which are probably more related to the range of intrahepatic metformin concentrations than those observed in plasma. We found that metformin inhibited cumulative glucose production stimulated by the cAMP analog dibutyryl-cAMP $\left(\mathrm{Bt}_{2}\right.$-CAMP) in mouse primary hepatocytes (Figure 1A), consistent with previous findings (8).
Interestingly, the ability of metformin to suppress $\mathrm{Bt}_{2}$-cAMP-stimulated glucose production was maintained in primary hepatocytes lacking both AMPK $\alpha 1$ and $-\alpha 2$ catalytic subunits (AMPK $\alpha 1 \alpha 2$-null hepatocytes) regardless of the gluconeogenic substrates used (lactate/pyruvate or dihydroxyacetone) (Figure 1A and Supplemental Figure 1; supplemental material available online with this article; doi:10.1172/JCI40671DS1). Furthermore, the inhibitory effect of metformin on both basal and cAMP-stimulated glucose production was observed as early as 4 hours after treatment in control and AMPK $\alpha 1 \alpha 2$-null hepatocytes (Supplemental Figure 2). Activation of the PKA signaling pathway by $\mathrm{Bt}_{2}$-cAMP was not impaired in the absence of AMPK or by metformin treatment, as demonstrated by the similarity of phospho-PKA substrate profiles in AMPK-deficient compared with control hepatocytes (Supplemental Figure 3). Of note, a high dose of metformin (>1 mM) slightly affected PKA signaling due to an adverse effect on hepatocytes (see below). Although basal glucose production in AMPK $\alpha 1 \alpha 2$-null hepatocytes tended to be lower compared with that in control hepatocytes (the difference was not statistically significant), $\mathrm{Bt}_{2}$-cAMP-stimulated glucose production was increased to levels similar to those in the controls (Figure $1 \mathrm{~A}$ and Supplemental Figures 1 and 2). However, it should be noted that the inhibitory action of metformin on hepatic glucose production was much more pronounced in AMPK-deficient hepatocytes, even at the lower doses (Figure 1A and Supplemental Figures 1 and 2). We then examined whether treatment of primary mouse hepatocytes with metformin increased AMPK phosphorylation at Thr172 and its downstream target acetyl-CoA carboxylase (ACC) at Ser79 in a dosedependent manner (Figure 1B). We observed that metformin treatment resulted in a slower mobility of total AMPK $\alpha$ on SDS-PAGE gels, which was consistent with increased Thr172 phosphorylation (Figure 1B). Moreover, PKA activation by $\mathrm{Bt}_{2}$-AMPc did not modify AMPK phosphorylation in hepatocytes (Figure 1B) unlike adipocytes (28). In AMPK $\alpha 1 \alpha 2$-null hepatocytes, expression of AMPK $\alpha$ was not detectable, and ACC phosphorylation was completely abolished with or without metformin, which was not due to changes in total ACC protein levels (Figure 1B).

We examined the key signaling step involved in the inhibition of glucose production by metformin by monitoring the phosphorylation of the transcriptional coactivator CRTC2, a key mediator controlling gluconeogenesis in the liver in response to fasting (18). Exposure of cultured primary hepatocytes to $\mathrm{Bt}_{2}$-cAMP triggered CRTC2 dephosphorylation, as judged by a faster electrophoretic mobility of total CRTC2 protein on SDS-PAGE, in both wild-type and AMPK-deficient hepatocytes (Figure 1B). Addition of increasing concentrations of metformin resulted in a slower migration of CRTC2, indicating enhanced phosphorylation (Figure 1B). The phosphorylation of CRTC2 was lost in AMPK $\alpha 1 \alpha 2$-null hepatocytes, demonstrating a role for AMPK in CRTC2 phosphorylation in response to metformin. However, despite a lack of AMPKinduced CRTC2 phosphorylation in AMPK $\alpha 1 \alpha 2$-null hepatocytes, metformin robustly inhibited glucose production (Figure 1A). These data indicate that metformin inhibits hepatic glucose production via AMPK- and CRTC2-independent mechanisms.

We measured the expression of the genes encoding the transcriptional coactivator PGC- $1 \alpha$ and key gluconeogenic enzymes including PEPCK and G6Pase to examine whether metformin modulates the gluconeogenic program in hepatocytes lacking AMPK. Under basal conditions, expression of Pgc-1 $\alpha$, G6Pase, and Pepck was similar in AMPK-deficient and control hepatocytes (Figure 1C), consistent with normal glucose production in AMPK $\alpha 1 \alpha 2$-null hepatocytes 
A

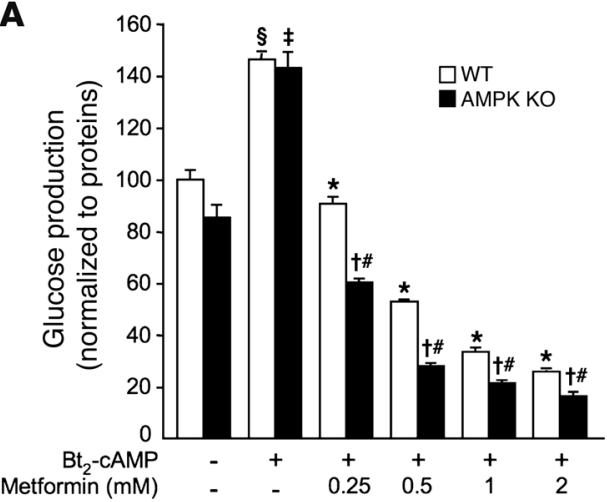

B

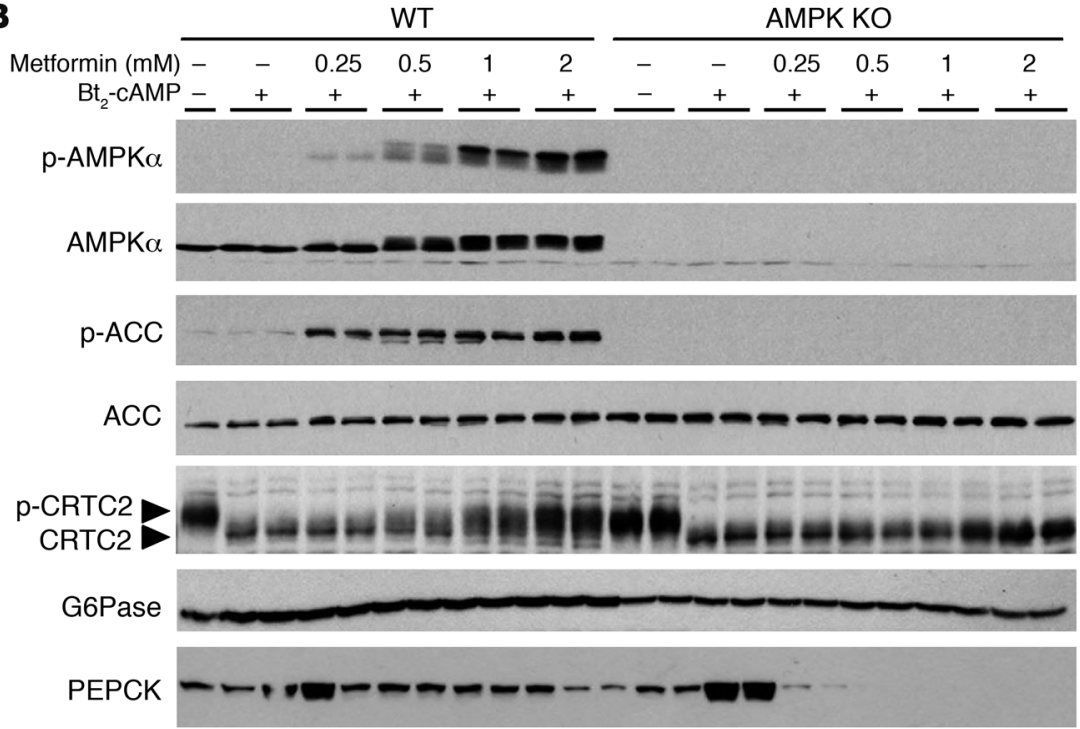

C

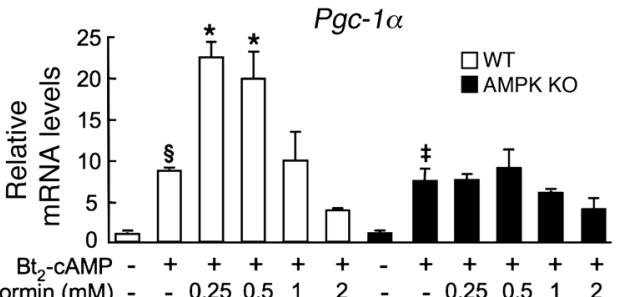

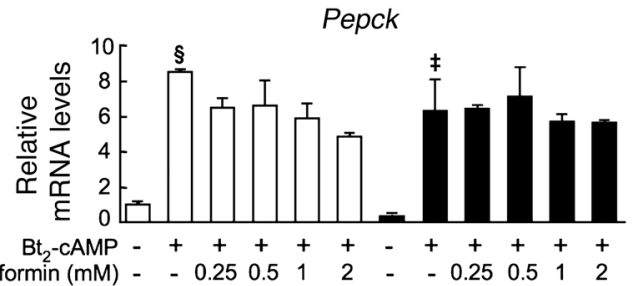

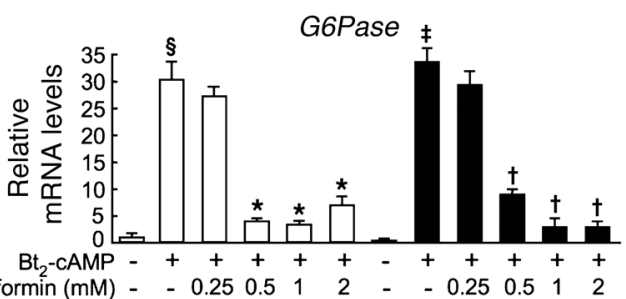

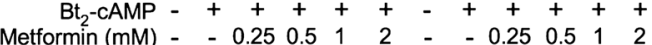

Figure 1

Metformin inhibits gluconeogenesis in AMPK $\alpha 1 \alpha 2$-null (AMPK KO) mouse hepatocytes. After attachment, WT and AMPK-deficient primary hepatocytes were cultured for 16 hours in M199 medium containing $100 \mathrm{nM}$ dex. Hepatocytes were then incubated in glucose-free DMEM containing lactate/pyruvate $(10: 1 \mathrm{mM})$ and $100 \mathrm{nM}$ dex alone or with $100 \mu \mathrm{M} \mathrm{Bt} \mathrm{t}_{2}-\mathrm{cAMP}$ and with or without $0.25,0.5,1$, or $2 \mathrm{mM}$ metformin. After 8 hours, medium was collected for glucose measurement and cells were harvested for Western blot and gluconeogenic gene expression analyses. (A) Glucose production was normalized to protein content and presented as a percentage of glucose produced by WT hepatocytes incubated in the absence of both $\mathrm{Bt}_{2}-\mathrm{CAMP}$ and metformin. Results are representative of 5 independent experiments. (B) Immunoblots were performed against phospho-AMPK $\alpha$ (Thr172), AMPK $\alpha$, phospho-ACC (Ser79), ACC, CRTC2, G6Pase, and PEPCK. Blots are representative of at least 5 independent experiments. (C) Relative mRNA levels of Pgc-1 $\alpha$, Pepck, and G6Pase expressed as fold activation relative to levels in WT hepatocytes incubated in the absence of both $\mathrm{Bt}_{2}-\mathrm{CAMP}$ and metformin. Results are representative of 5 independent experiments. Data are mean $\pm \mathrm{SEM}$. $\$ P<0.001, \ddagger P<0.001$ compared with WT and AMPK-KO hepatocytes incubated without $\mathrm{Bt}_{2}$-CAMP; ${ }^{*} P<0.001,+P<0.001$ compared with WT and AMPK-KO hepatocytes incubated with $\mathrm{Bt}_{2}$-CAMP alone; ${ }^{\#} P<0.01$ compared with WT hepatocytes incubated under the same conditions.

(Figure 1A). Bt $t_{2}$-cAMP increased the expression of these genes to similar levels in both AMPK $\alpha 1 \alpha 2$-null and control hepatocytes (Figure 1C). Following metformin treatment, expression of gene encoding PGC-1 $\alpha$ was increased more in control hepatocytes than in AMPK $\alpha 1 \alpha 2$-null hepatocytes, indicating that $P g c-1 \alpha$ gene expression is controlled by an AMPK-dependent mechanism (Figure 1C). Metformin had differential effects on the expression of the genes encoding the gluconeogenic enzymes G6Pase and PEPCK, although it suppressed glucose production. While metformin inhibited $\mathrm{Bt}_{2}$-cAMP-stimulated G6Pase gene expression in a concentration- dependent manner, it had only a marginal effect on Pepck gene expression, even at the highest concentration of metformin (Figure 1C). Similar gene expression patterns for G6Pase and Pepck were observed in AMPK $\alpha 1 \alpha 2$-null hepatocytes treated with metformin (Figure 1C). Changes in PEPCK and G6Pase protein levels were not consistent with the gene transcription data. In control hepatocytes, the amount of G6Pase was increased by $\mathrm{Bt}_{2}$-cAMP and remained constant despite increasing metformin concentrations (Figure 1B) and severe G6Pase gene repression (Figure 1C). In AMPK $11 \alpha 2$-null hepatocytes, G6Pase protein levels were lower than those in wild- 

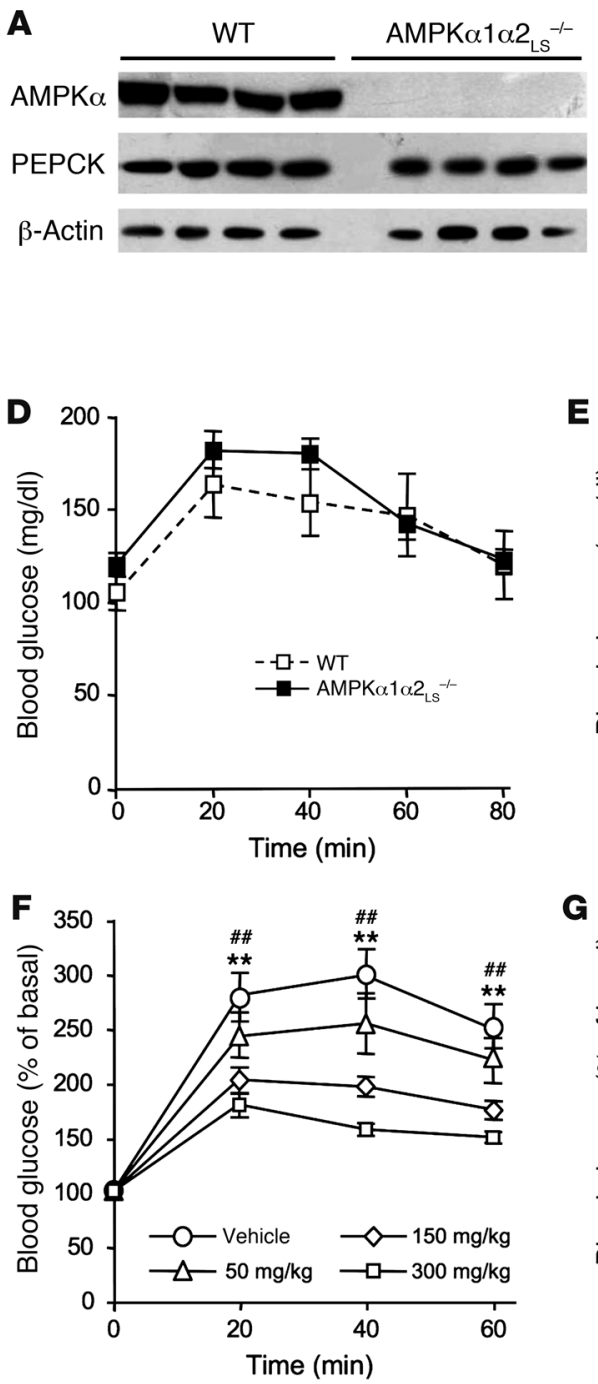

G
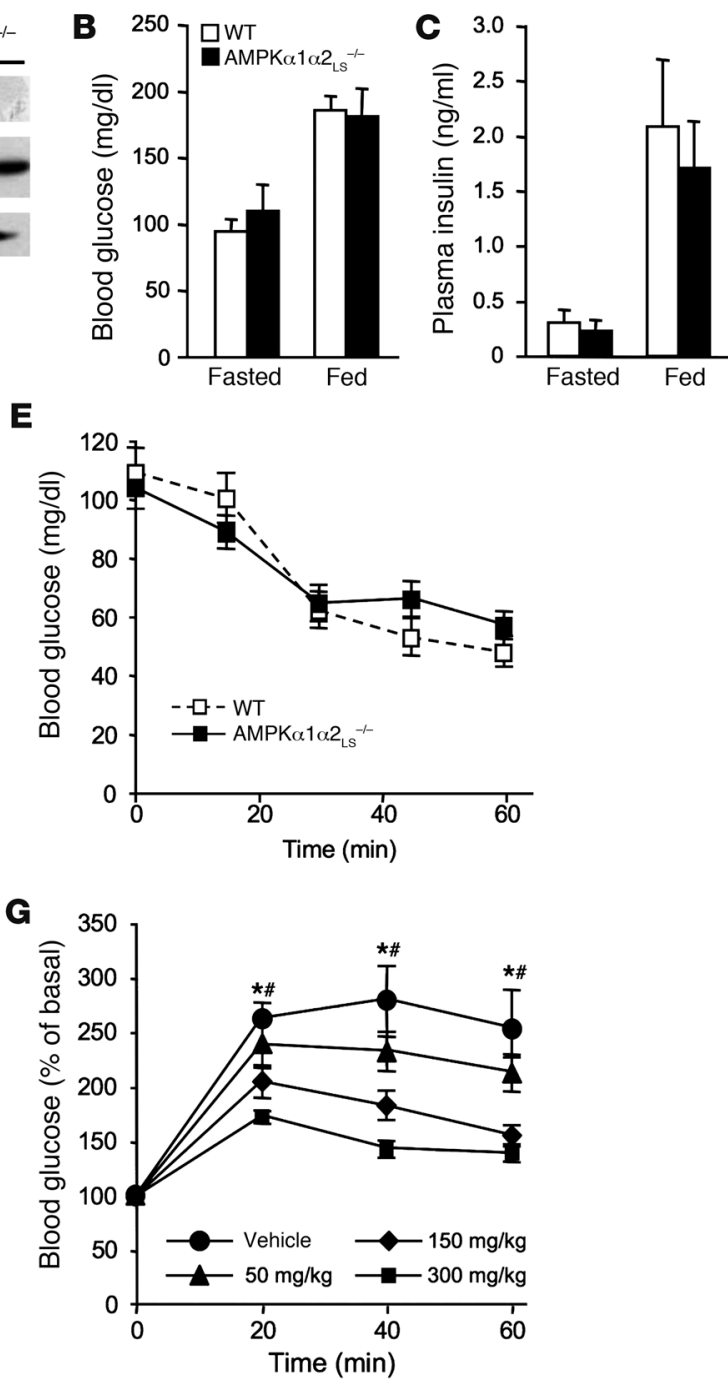

Figure 2

Effects of metformin on blood glucose levels in AMPK $\alpha 1 \alpha 2 \mathrm{LS}^{-1-}$ mice. (A) Western blot analysis of AMPK $\alpha$ and PEPCK proteins in livers from 24-hour-fasted control and AMPK $\alpha 1 \alpha 2 \mathrm{LS}^{-/-}$mice. $\beta$-Actin was immunoblotted as a loading control. Each lane represents the liver sample from an individual mouse. (B) Plasma blood glucose levels were measured in fasted and fed control and AMPK $\alpha 1 \alpha 2 \mathrm{LS}^{-1-}$ mice. $n=5-6$. (C) Plasma insulin levels were measured in fasted and fed control and AMPK $\alpha 1 \alpha 2 \mathrm{LS}^{-/-}$mice. Data are mean \pm SEM $(n=5-6)$. (D) Pyruvate tolerance tests $(2 \mathrm{~g} / \mathrm{kg})$ in control and AMPK $\alpha 1 \alpha 2 \mathrm{LS}^{-1-}$ mice were used to assess hepatic gluconeogenesis. $n=6-7$. (E) Insulin tolerance tests $(0.25 \mathrm{U} / \mathrm{kg})$ in control and AMPK $\alpha 1 \alpha 2 \mathrm{LS}^{-/-}$mice. $n=6-9$. Metformin tolerance tests in control (F) and AMPK $\alpha 1 \alpha 2 \mathrm{Ls}^{-/-}$ (G) mice. Mice were given an oral gavage dose of 50,150 , or $300 \mathrm{mg} / \mathrm{kg}$ metformin or vehicle and after 30 minutes challenged with an oral administration of glucose $(3 \mathrm{~g} / \mathrm{kg}$ body weight). $n=6-10$. Data are mean \pm SEM. ${ }^{\star} P<0.05,{ }^{* \star} P<0.005,150 \mathrm{mg} / \mathrm{kg}$ metformin compared with vehicle control; $\# P<0.01$, \# $P<0.001$, $300 \mathrm{mg} / \mathrm{kg}$ compared with the vehicle control. type hepatocytes and were unaffected by $\mathrm{Bt}_{2}$-cAMP or metformin treatment (Figure 1B). In contrast to unaltered Pepck gene transcription, PEPCK protein levels were markedly decreased at all doses of metformin in WT hepatocytes, and a complete loss of PEPCK protein was observed in AMPK $\alpha 1 \alpha 2$-null hepatocytes (Figure 1B).

Metformin lowers blood glucose levels in liver AMPK-deficient mice. The principal mechanism by which metformin lowers blood glucose levels in vivo is the suppression of hepatic glucose production (6). Therefore, to investigate the role of AMPK in metformin action, we used mice in which both AMPK catalytic subunits had been deleted in the liver (AMPK $\alpha 1 \alpha 2_{\mathrm{LS}^{-/-}}$mice; ref. 29). Expression of AMPK $\alpha 1 \alpha 2$ proteins was undetectable in livers of AMPK $\alpha 1 \alpha 2_{\mathrm{LS}^{-/-}}$ mice (Figure 2A). Fed and fasting blood glucose levels were similar in AMPK $\alpha 1 \alpha 2_{\mathrm{LS}^{-/-}}$and control mice, as were plasma insulin levels (Figure 2, B and C), suggesting normal regulation of glucose homeostasis in these mice. We then determined the role of AMPK in modulating gluconeogenesis in vivo by examining blood glucose levels in mice following intraperitoneal injection of pyruvate, a major gluconeogenic substrate. Blood glucose concentrations increased in both AMPK $\alpha 1 \alpha 2_{\mathrm{LS}}^{-/-}$and control mice, although the differences were not significant (Figure 2D). These results indicate that $\mathrm{AMPK} \alpha 1 \alpha 2_{\mathrm{LS}^{-/-}}$mice are as capable of converting pyruvate to glucose as normal mice, as suggested by comparable levels of the key gluconeogenic enzyme PEPCK in both groups of mice (Figure $2 \mathrm{~A})$. Moreover, AMPK $\alpha 1 \alpha 2_{\mathrm{LS}^{-/-}}$and control mice displayed normal responses to insulin during insulin tolerance tests, indicating unaffected hepatic insulin sensitivity in AMPK $\alpha 1 \alpha 2_{\mathrm{LS}^{-/-}}$mice (Figure 2E). During oral glucose tolerance tests, blood glucose excursion was markedly increased in AMPK $\alpha 1 \alpha 2_{\mathrm{LS}^{-/-}}$and control mice (Figure 2, F and G), with equivalent increases in plasma insulin levels 20 minutes after glucose load $(0.96 \pm 0.29 \mathrm{ng} / \mathrm{ml}$ insulin in AMPK $\alpha 1 \alpha 2_{\mathrm{LS}^{-/-}}$mice versus $0.97 \pm 0.24 \mathrm{ng} / \mathrm{ml}$ insulin in WT mice; $n=4-6)$. Next, to investigate metformin action in vivo, we examined control and liver AMPK-deficient mice after intragastric administration of increasing doses of metformin $(50,150$, and $300 \mathrm{mg} / \mathrm{kg})$ and glucose. These relatively high doses of metformin are necessary in rodents to reach plasma metformin concentrations similar to those found in humans and to produce a therapeutic effect in diabetic animals (30). Oral administration of metformin caused a significant reduction in blood glucose excursion in a dose-dependent manner in control (Figure 2F) but also in AMPK $\alpha 1 \alpha 2_{\mathrm{LS}^{-/-}}$mice (Figure 2G). Plasma insulin levels 20 minutes after glucose load were similar in animals treated with $300 \mathrm{mg} / \mathrm{kg}$ metformin $(0.63 \pm 0.13 \mathrm{ng} / \mathrm{ml}$ insulin in AMPK $\alpha 1 \alpha 2_{\mathrm{LS}^{-/-}}$mice versus $0.72 \pm 0.32 \mathrm{ng} / \mathrm{ml}$ insulin in WT 
A
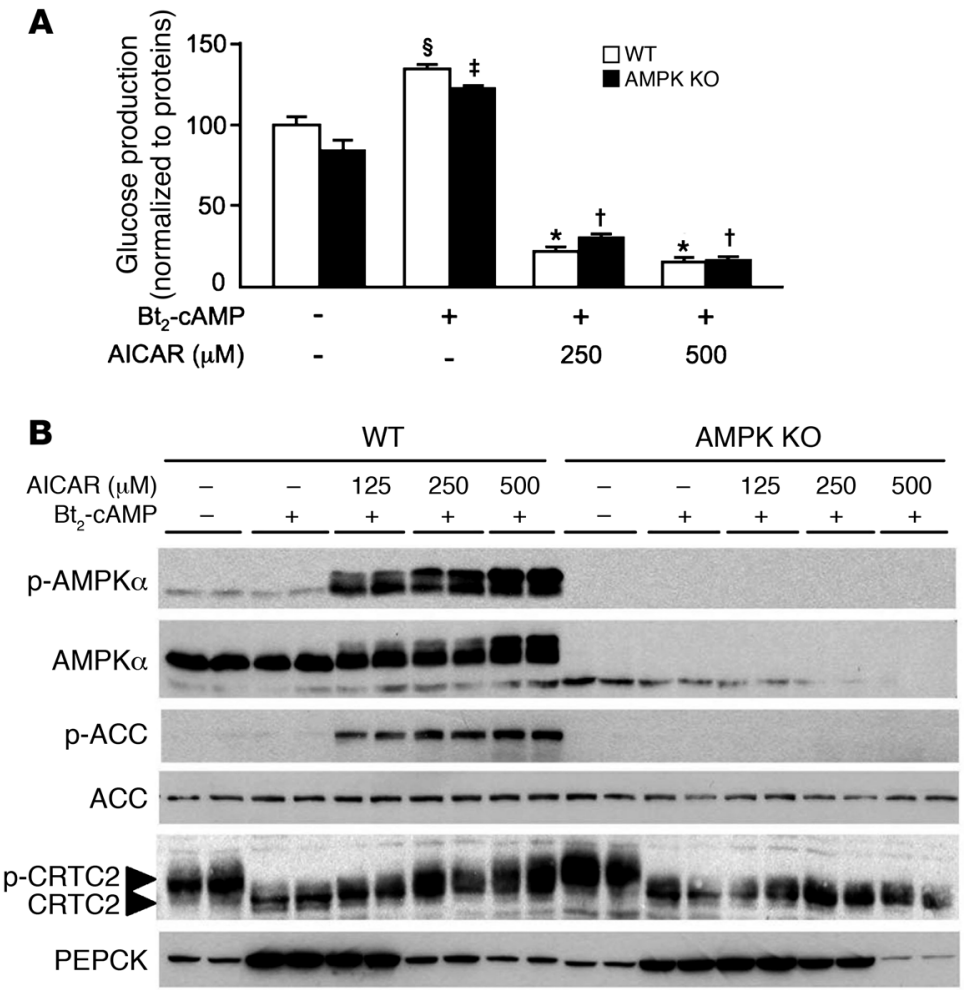
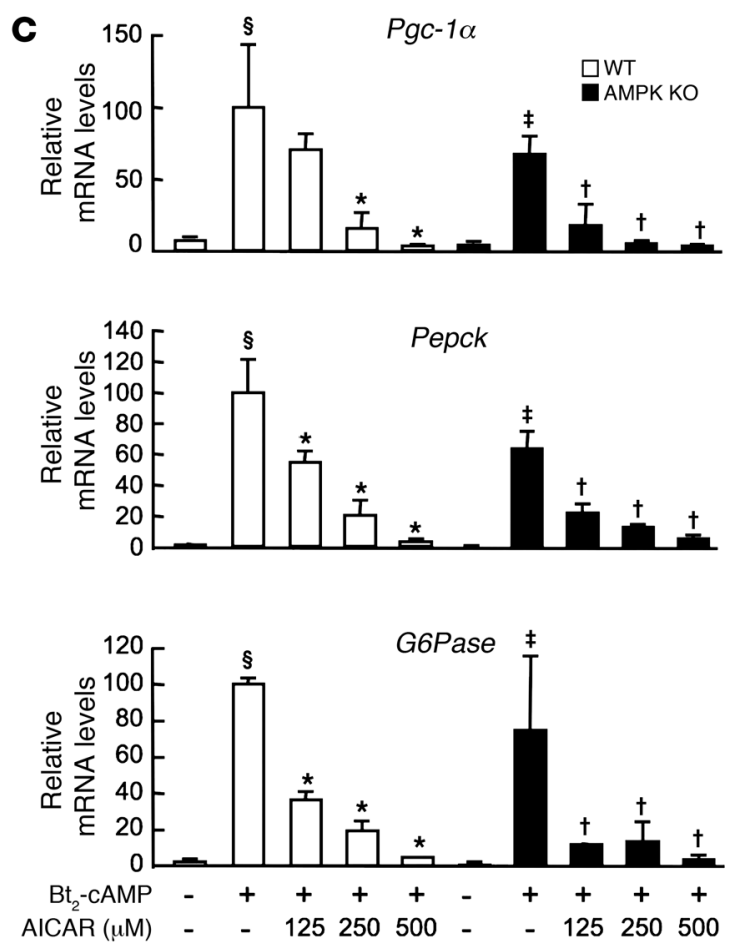

\section{Figure 3}

Effects of AICAR on gluconeogenesis in WT and AMPK-KO hepatocytes. After attachment, WT and AMPK-deficient primary hepatocytes were cultured for 16 hours in M199 medium containing $100 \mathrm{nM}$ dex. Hepatocytes were then incubated in glucose-free DMEM containing lactate/pyruvate $(10: 1 \mathrm{mM})$ and $100 \mathrm{nM}$ dex, alone or with $100 \mu \mathrm{M} \mathrm{Bt}_{2}$-CAMP and with or without 125, 250, or $500 \mu \mathrm{M}$ AICAR. After 8 hours, medium was collected for glucose measurement, and cells were harvested for Western blot and gluconeogenic gene expression analyses. (A) Glucose production was normalized to protein content and expressed as a percentage of glucose production by WT hepatocytes incubated in the absence of both $\mathrm{Bt}_{2}$-CAMP and AICAR. Results are representative of 5 independent experiments. (B) Immunoblots were performed against phospho-AMPK $\alpha$ (Thr172), AMPK $\alpha$, phospho-ACC (Ser79), ACC, CRTC2, and PEPCK. Blots are representative of at least 3 independent experiments. (C) Relative mRNA levels of $P g c-1 \alpha, P e p c k$, and G6Pase expressed as a percentage of WT hepatocytes incubated in the absence of both Bt ${ }_{2}-\mathrm{CAMP}_{\mathrm{M}}$ and AICAR. Results are representative of 5 independent experiments. Data are mean \pm SEM. $\$ P<0.01, \neq P<0.01$ compared with WT and AMPK-KO hepatocytes incubated without $\mathrm{Bt}_{2}-\mathrm{CAMP} ;{ }^{*} P<0.01,{ }^{\dagger} P<0.01$ compared with WT and AMPK-KO hepatocytes incubated with $\mathrm{Bt}_{2}-\mathrm{CAMP}$ alone.

mice; $n=4-5)$. The improvement in glucose tolerance by metformin treatment was similar in AMPK $\alpha 1 \alpha 2_{\mathrm{LS}^{-/-}}$and control mice, indicating equivalent sensitivity to metformin. These results are consistent with the data from primary hepatocyte culture and further support the notion that metformin action on hepatic glucose production is AMPK independent.

Control of hepatic gluconeogenesis by AICAR and A-769662 in AMPKdeficient hepatocytes. We further explored the molecular mechanisms by which AMPK activators regulate gluconeogenesis by stimulating $\mathrm{AMPK} \alpha 1 \alpha 2$-null and control hepatocytes with $\mathrm{Bt}_{2}$-cAMP in the presence or absence of increasing amounts of AICAR (5-aminoimidazole-4-carboxamide-1- $\beta$-D-ribofuranoside) - a cell-permeable compound that is phosphorylated to AICAR monophosphate (ZMP), a cellular mimetic of AMP (31) - and a small-molecule thienopyridone, A-769662, that directly activates AMPK via an AMP-independent mechanism (32-35). Treatment with AICAR strongly inhibited glucose production in both AMPK $\alpha 1 \alpha 2$-null and control hepatocytes (Figure 3A). In contrast, A-769662 had no significant effect on glucose production in AMPK $\alpha 1 \alpha 2$-null and control hepatocytes (Figure 4A). The reduction of glucose production observed at high A-769662 concentration in both
AMPK $\alpha 1 \alpha 2$-null and control hepatocytes probably reflects nonspecific effects caused by this compound at concentrations higher than $100 \mu \mathrm{M}$ (M. Foretz, unpublished observations, and ref. 36). Treatment with AICAR induced a dose-dependent increase in the phosphorylation of AMPK and its downstream targets ACC and CRTC2 in control hepatocytes, whereas phosphorylation of these enzymes was completely abolished in AMPK $\alpha 1 \alpha 2$-null hepatocytes (Figure 3B). In contrast, treatment with A-769662 did not change CRTC2 phosphorylation status (Figure 4B). This may be due to selective action of the compound on specific AMPK heterotrimers, as recently reported (35). Consistent with the above results on glucose production (Figure $3 \mathrm{~A}$ and Figure $4 \mathrm{~A}$ ), AICAR inhibited $\mathrm{Bt}_{2}$ cAMP-stimulated expression of genes encoding PGC-1 $\alpha$, G6Pase, and PEPCK (Figure 3C) whereas A-769662 had no effect on gluconeogenic gene expression (Figure 4C).

Although A-769662 stimulated phosphorylation of ACC at levels equivalent to AICAR, phosphorylation of AMPK was fairly modest (Figure 4B). It was therefore not clear whether AMPK Thr172 phosphorylation was required for A-769662 action. To address this, we monitored ACC phosphorylation in hepatocytes lacking LKB1, the upstream kinase involved in the phosphorylation 

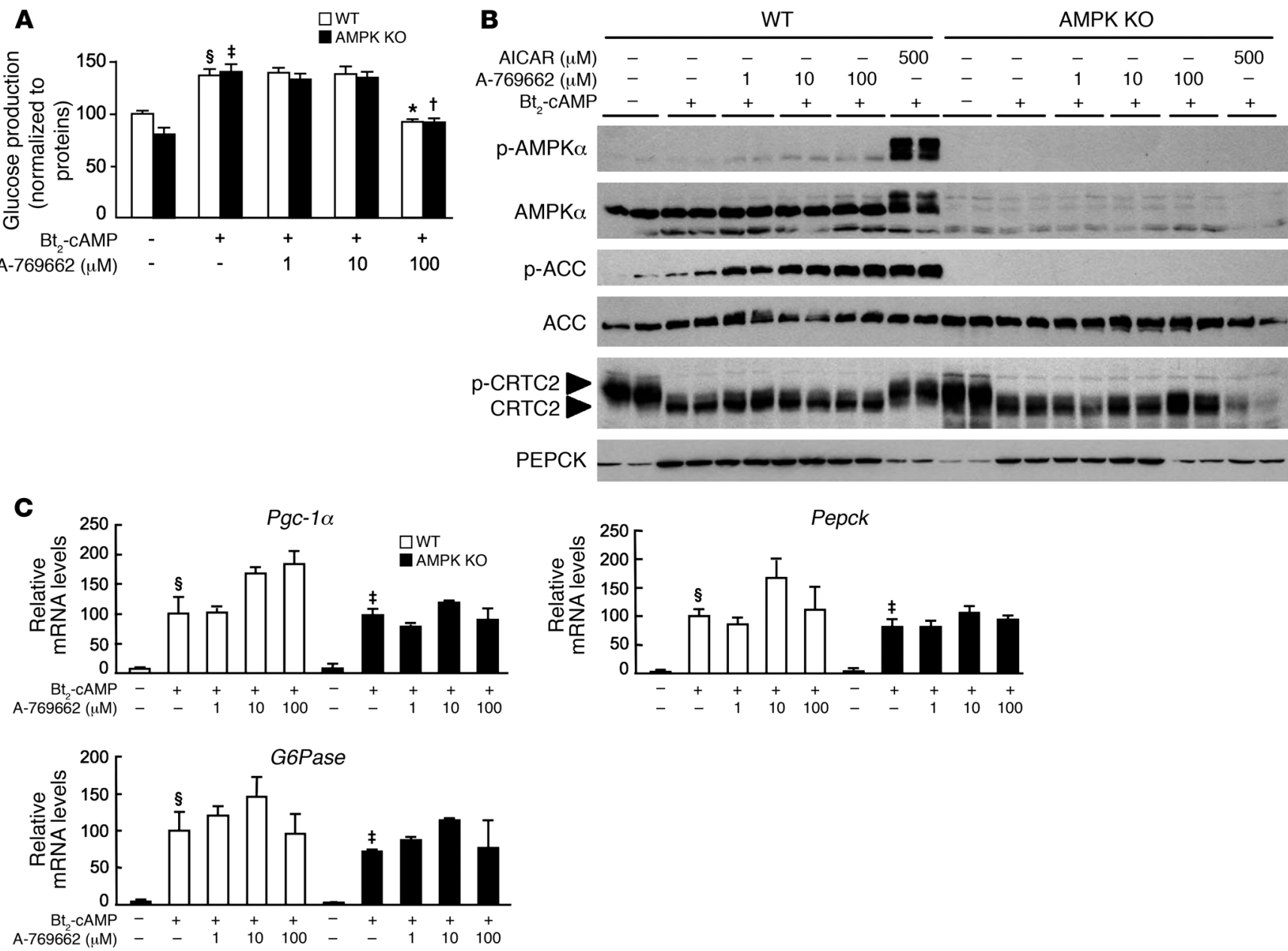

\section{Figure 4}

Effects of A-769662 on gluconeogenesis in WT and AMPK-KO hepatocytes. After attachment, WT and AMPK-deficient primary hepatocytes were cultured for 16 hours in M199 medium containing $100 \mathrm{nM}$ dex. Hepatocytes were then incubated in glucose-free DMEM containing lactate/pyruvate $(10: 1 \mathrm{mM})$ and $100 \mathrm{nM}$ dex alone or with $100 \mu \mathrm{M} \mathrm{Bt}_{2}$-CAMP and with or without 1, 10, or $100 \mu \mathrm{M} \mathrm{A}-769662$. After 8 hours, medium was collected for glucose measurement and cells were harvested for Western blot and gluconeogenic gene expression analyses. (A) Glucose production was normalized to protein content and expressed as a percentage of glucose production by WT hepatocytes incubated in the absence of both $\mathrm{Bt}_{2}$-cAMP and A-769662. Results are representative of 5 independent experiments. (B) Immunoblots were performed against phospho-AMPK $\alpha$ (Thr172), AMPK $\alpha$, phospho-ACC (Ser79), ACC, CRTC2, and PEPCK. Blots are representative of least 5 independent experiments. (C) Relative mRNA levels of $P g c-1 \alpha, P e p c k$, and G6Pase expressed as fold activation relative to levels in WT hepatocytes incubated in the absence of both Bt ${ }_{2}-\mathrm{CAMP}_{\mathrm{M}}$ and A-769662. Results are representative of 5 independent experiments. Data are mean \pm SEM. $\$ P<0.01, \ddagger P<0.01$ compared with WT and AMPK-KO hepatocytes incubated without $\mathrm{Bt}_{2}-\mathrm{CAMP} ;{ }^{*} P<0.01,{ }^{\dagger} P<0.01$ compared with WT and AMPK-KO hepatocytes incubated with $\mathrm{Bt}_{2}-\mathrm{CAMP}$ alone.

of Thr172 within the activation loop of the $\alpha$ catalytic subunit kinase domain. In the absence of LKB1, A-769662 was unable to induce ACC phosphorylation (Supplemental Figure 4), indicating that LKB1-mediated AMPK phosphorylation was necessary for A-769662 action in hepatocytes. Recent studies demonstrated that A-769662 mimics the effects of AMP on the AMPK system, but via an AMP-independent mechanism, suggesting that A-769662 binds to an alternate allosteric site (32-34). We investigated this further by evaluating the additive effect of AICAR and A-769662 on AMPK phosphorylation in hepatocytes. In the presence of a saturating concentration of A-769662 $(30 \mu \mathrm{M})$, AICAR-induced AMPK phosphorylation was further increased, demonstrating a synergic action of these two compounds on AMPK activation (Supplemental Figure 5), reflecting A-769662 protection against Thr172 dephosphorylation (34). Similarly, AICAR-induced phos- phorylation of AMPK downstream targets ACC and CRTC2 was enhanced in the presence of A-769662 (Supplemental Figure 5).

Metformin affects hepatic energy state. Gluconeogenesis is an energydemanding process, consuming 4 ATP and 2 GTP molecules per molecule of glucose produced. It has been demonstrated that metformin inhibits complex I of the mitochondrial respiratory chain in isolated hepatocytes $(37,38)$, increasing the cellular AMP/ATP ratio, which in turn activates AMPK (29). In order to evaluate the regulatory potential of metformin on hepatic energy charge, we assessed adenine nucleotide content in primary hepatocytes isolated from $\mathrm{C} 57 \mathrm{BL} / 6 \mathrm{~J}$ mice following treatment with increasing doses of metformin and in the presence or absence of $\mathrm{Bt}_{2}$-cAMP. Stimulation of hepatocytes with $\mathrm{Bt}_{2}$-cAMP increased cellular ATP content (Figure 5A), as previously described (39). ATP levels were decreased by metformin in a concentration-dependent fashion in both basal 

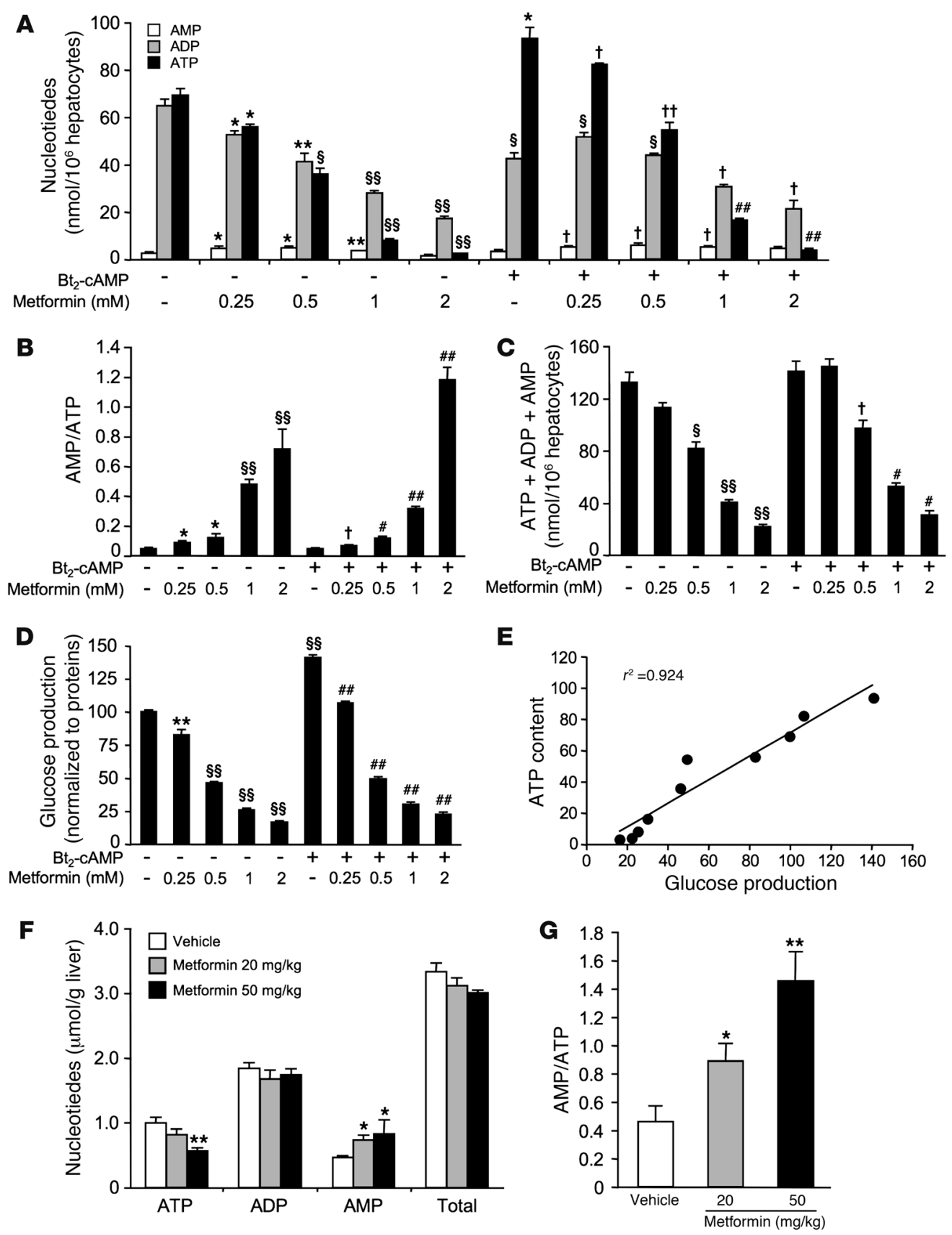

Figure 5

Metformin reduces energy state in primary hepatocytes and in liver of C57BL/6J mice. (A-E) After attachment, C57BL/6J mouse primary hepatocytes were cultured for 16 hours in M199 medium containing $100 \mathrm{nM}$ dex. Hepatocytes were then incubated in glucose-free DMEM containing lactate/pyruvate $(10: 1 \mathrm{mM})$ and $100 \mathrm{nM}$ dex alone or with $100 \mu \mathrm{M} \mathrm{Bt}_{2}$-cAMP and with or without $0.25,0.5,1$, or $2 \mathrm{mM}$ metformin. After 8 hours, medium was collected for glucose measurement and cells were harvested for measurement of adenine nucleotide content. (A) ATP, ADP, and AMP content in hepatocytes, (B) AMP/ATP ratios, and (C) total adenine nucleotide content are shown for each condition. (D) Glucose production was normalized to protein content and presented as a percentage of glucose produced by hepatocytes incubated in the absence of both $\mathrm{Bt}_{2}$-cAMP and metformin. (E) Correlation between glucose production and ATP content shown in $\mathbf{D}$ and $\mathbf{A}$, respectively. Results are representative of 3 independent experiments. ${ }^{*} P<0.05$, ${ }^{*} P<0.01, \S P<0.005$, $\S \S P<0.001$ compared with hepatocytes incubated in the absence of both $\mathrm{Bt}_{2}-\mathrm{cAMP}$ and metformin; ${ }^{\dagger} P<0.05,{ }^{\dagger} P<0.01, \# P<0.005$, \#\# $P<0.001$ compared with hepatocytes incubated with $\mathrm{Bt}_{2}$-cAMP alone. ( $\mathbf{F}$ and $\mathbf{G}$ ) Ten-week-old C57BL/6J male mice ( $n=7$ per group) were treated orally with 20 or $50 \mathrm{mg} / \mathrm{kg}$ metformin in water or with water alone for 5 consecutive days. On the fifth day, mice were fasted for 24 hours and liver was collected 1 hour after metformin administration for hepatic ATP, ADP, and AMP determination. (F) Total adenine nucleotide content and (G) AMP/ATP ratios are shown for each condition. ${ }^{\star} P<0.05,{ }^{\star \star} P<0.005$ compared with vehicle. Data are mean \pm SEM. 


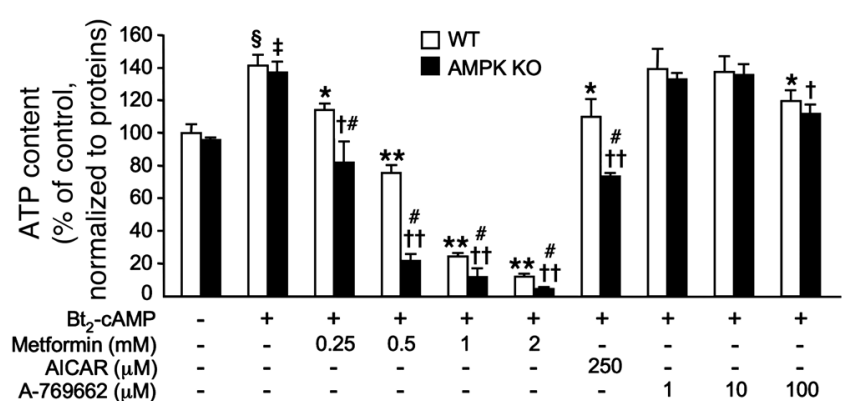

Figure 6

Effects of AMPK activators on intracellular ATP content in WT and AMPK-KO hepatocytes. After attachment, WT and AMPK-deficient primary hepatocytes were cultured for 16 hours in M199 medium containing $100 \mathrm{nM}$ dex. Hepatocytes were then incubated in glucose-free DMEM containing lactate/pyruvate $(10: 1 \mathrm{mM})$ and $100 \mathrm{nM}$ dex alone or with $100 \mu \mathrm{M} \mathrm{Bt}_{2}$-CAMP and with or without various concentrations of metformin, AICAR, or A-769662 as indicated. After 8 hours, cells were harvested for ATP content measurement. Results are representative of 6 independent experiments. Data are mean \pm SEM. $\$ P<0.001$, $\ddagger P<0.001$ compared with WT and AMPK-KO hepatocytes incubated without $\mathrm{Bt}_{2}$-CAMP; ${ }^{\star} P<0.05,{ }^{\star \star} P<0.001$ compared with WT hepatocytes incubated with $\mathrm{Bt}_{2}$-CAMP alone; ${ }^{\dagger} P<0.01$, ${ }^{\dagger+} P<0.001$ compared with AMPK-KO hepatocytes incubated with $\mathrm{Bt}_{2}$-CAMP alone; $\# P<0.01$ compared with WT hepatocytes incubated under the same conditions.

and cAMP-stimulated conditions (Figure 5A), consistent with our previous findings (29). Conversely, AMP levels were increased with metformin, leading to a significant increase in the AMP/ATP ratio in both basal and cAMP-stimulated conditions (Figure 2B). However, it should be noted that there was a significant reduction in total adenine nucleotide content for the highest metformin concentrations (Figure 5C), indicating an adverse effect of high doses of metformin on primary hepatocytes. Interestingly, the decrease in intracellular ATP content with metformin treatment (Figure $5 \mathrm{~A})$ precisely paralleled the inhibition pattern of metformin on glucose production (Figure 5D). Therefore, a clear relationship $\left(r^{2}=0.924\right)$ exists between the inhibitory effect of metformin on glucose production and hepatic ATP content (Figure 5E).

To further investigate the effect of metformin on hepatic energy charge in vivo, liver adenine nucleotides were examined in C57BL/6J mice after intragastric administration of low doses of metformin (20 and $50 \mathrm{mg} / \mathrm{kg}$ ). It has been previously reported that in mice treated with $50 \mathrm{mg} / \mathrm{kg}$ metformin, plasma metformin concentrations peaked at 52 and $29 \mu \mathrm{M}$ in the hepatic portal vein and the inferior vena cava, respectively (26), and are similar to those found therapeutically in humans (25). In response to metformin administration, hepatic ATP, ADP, and AMP levels were decreased, unchanged, and increased, respectively, resulting in a 2- to 3-fold increase in AMP/ATP ratio compared with control mice (Figure 5, F and G). In addition, total adenine nucleotide content in the liver was unchanged at either concentration of metformin (Figure 5F). Metformin administration lowered hepatic energy charge from $0.57 \pm 0.01$ in vehicle-treated mice to $0.51 \pm 0.02(P<0.05)$ and $0.46 \pm 0.02(P<0.005)$ in $20 \mathrm{mg} / \mathrm{kg}$ and $50 \mathrm{mg} / \mathrm{kg}$ metformin-treated mice, respectively.

Given that metformin treatment is associated with changes in hepatic energy charge, we hypothesized that metformin inhibits hepatic glucose production in both wild-type and AMPK-deficient hepatocytes through the inhibition of ATP production. Stimulation of hepatocytes with $\mathrm{Bt}_{2}$-cAMP increased cellular ATP content in AMPK-deficient hepatocytes to levels similar to those in the controls (Figure 6). Cellular ATP content decreased in a dose-dependent manner when control hepatocytes were incubated with metformin and also AICAR (Figure 6), but the AICAR effect was less pronounced than that with metformin, as previously described $(29,40)$. Consistent with previous reports, A-769662 treatment resulted in no significant alteration in cellular ATP levels (Figure 6 and ref. 32).
A

\section{Figure 7}

Effects of metformin on AMPK activation in WT and $L k b 1-K O$ hepatocytes. After attachment, WT and LKB1-deficient primary hepatocytes were cultured for 16 hours in M199 medium containing $100 \mathrm{nM}$ dex. Hepatocytes were then incubated in glucose-free DMEM containing lactate/pyruvate $(10: 1 \mathrm{mM})$ and $100 \mathrm{nM}$ dex alone or with $100 \mu \mathrm{M}$ $\mathrm{Bt}_{2}$-cAMP and with or without $0.25,0.5,1$, or $2 \mathrm{mM}$ metformin. After 8 hours, cells were harvested for Western blot analysis and measurement of LKB1 activity. (A) The level of LKB1 protein was assessed by immunoblot analysis using antiLKB1 antibodies. $\beta$-Actin was immunoblotted as a loading control. (B) LKB1 activity was assessed following its immunoprecipitation and assayed with the LKBtide peptide. Assays were performed on hepatocyte extracts from 3 independent experiments. (C) Immunoblots were performed against phospho-AMPK $\alpha$ (Thr172), AMPK $\alpha$, phospho-ACC (Ser79), ACC, CRTC2, G6Pase, and PEPCK. Blots are representative of 3 independent experiments.
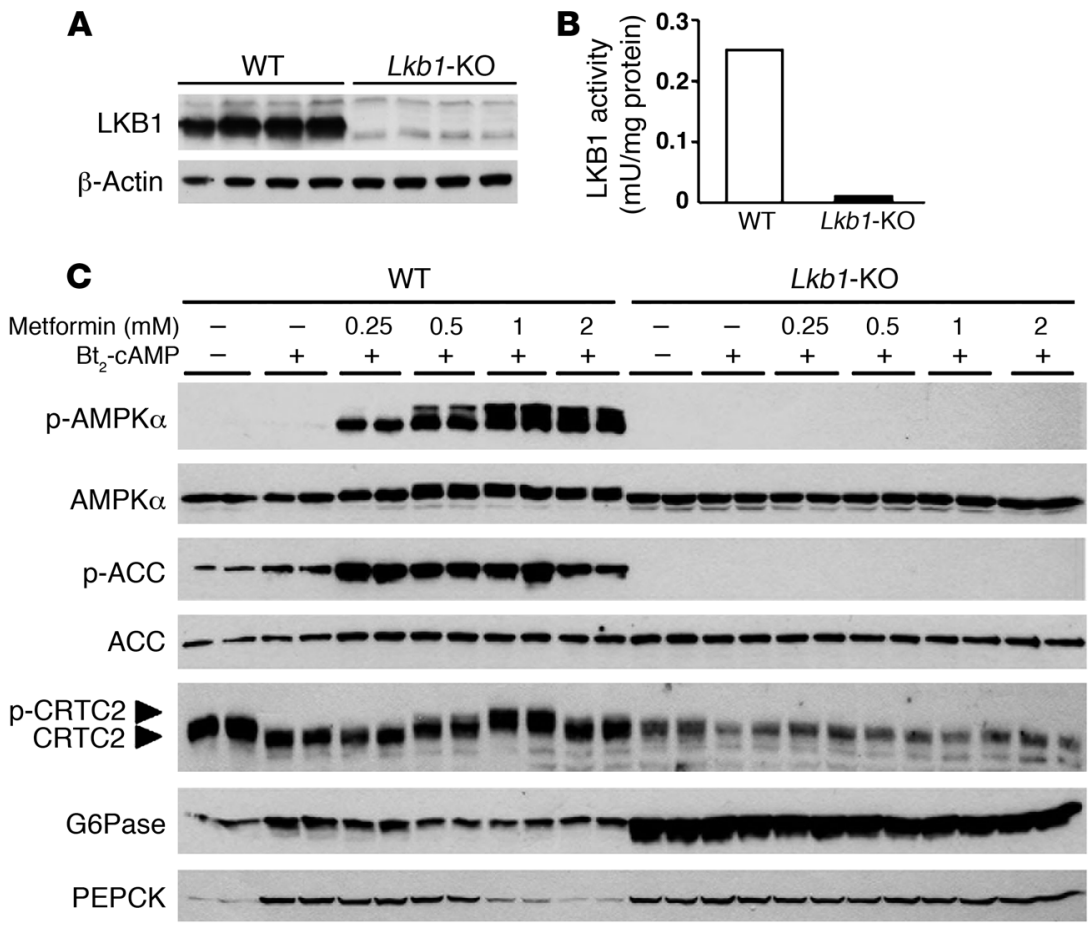

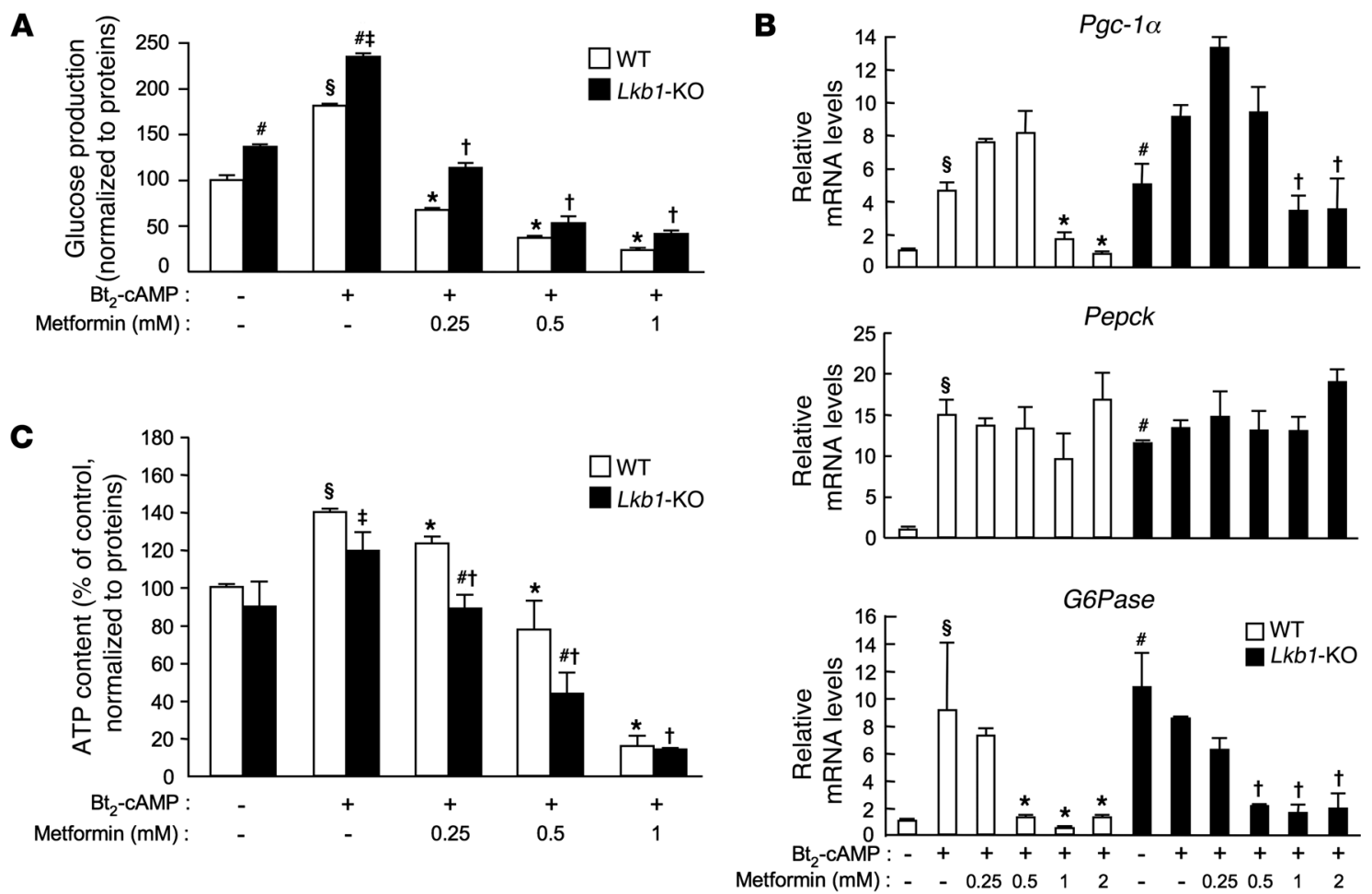

\section{Figure 8}

Metformin inhibits gluconeogenesis in LKB1-deficient mouse hepatocytes. After attachment, WT and LKB1-deficient primary hepatocytes were cultured for 16 hours in M199 medium containing $100 \mathrm{nM}$ dex. Hepatocytes were then incubated in glucose-free DMEM containing lactate/pyruvate $(10: 1 \mathrm{mM})$ and $100 \mathrm{nM}$ dex alone or with $100 \mu \mathrm{M} \mathrm{Bt}_{2}$-cAMP and with or without $0.25,0.5,1$, or $2 \mathrm{mM}$ metformin. After 8 hours, medium was collected for glucose measurement and cells were harvested for ATP content assessment and gluconeogenic gene expression analysis. (A) Glucose production was normalized to protein content and expressed as a percentage of glucose produced by WT hepatocytes incubated in the absence of both $\mathrm{Bt}_{2}$-cAMP and metformin. Results are representative of 3 independent experiments. (B) Relative mRNA levels of $P g c-1 \alpha, P e p c k$, and $G 6 P a s e$ expressed as fold activation relative to levels in WT hepatocytes incubated in the absence of both $\mathrm{Bt}_{2}$-cAMP and metformin. Results are representative of 3 independent experiments. (C) ATP intracellular content normalized to protein content and expressed as a percentage of WT hepatocyte ATP content incubated in the absence of both $\mathrm{Bt}_{2}$-CAMP and metformin. Results are representative of 4 independent experiments. Data are mean \pm SEM. $\$ P<0.01, \ddagger P<0.01$ compared with WT and AMPK-KO hepatocytes incubated without $\mathrm{Bt}_{2}$-CAMP; ${ }^{*} P<0.01,{ }^{\dagger} P<0.01$ compared with WT and $L k b 1-K O$ hepatocytes incubated with $\mathrm{Bt}_{2}$-cAMP alone; ${ }^{2} P<0.05$ compared with WT hepatocytes incubated under the same conditions.

However, at concentrations greater than $100 \mu \mathrm{M}$, ATP levels were slightly decreased in both control and AMPK-deficient hepatocytes, probably due to unknown side effects (M. Foretz, unpublished observations, and ref. 36). When AMPK-deficient hepatocytes were treated with metformin or AICAR, ATP levels dropped considerably more than in control hepatocytes (Figure 6), indicating the crucial role of AMPK in restoring the intracellular energy balance following reduction in ATP levels. Together, these results suggest that the effect of metformin on hepatocytes is linked to changes in intracellular ATP content.

Metformin suppresses hepatic gluconeogenesis in the absence of LKB1. We investigated the role of LKB1 in the action of metformin on hepatic glucose production by using hepatocytes from liver-specific Lkb1-KO mice. The Lkb1 gene was deleted in adult mouse liver by injection of an adenovirus expressing Cre recombinase in Lkb1-floxed mice (41). Western blot analysis confirmed a complete loss of LKB1 expression in hepatocyte primary cultures from liverspecific Lkb1-KO mice (Figure 7A). Accordingly, no LKB1 activity was detected in LKB1-deficient hepatocytes (Figure 7B). To determine whether lack of LKB1 affected AMPK activation, we treated
LKB1-deficient and control hepatocytes with increasing concentrations of metformin. Metformin robustly increased AMPK phosphorylation at Thr172, the site of LKB1 phosphorylation, as well as ACC phosphorylation at Ser79 in control hepatocytes (Figure 7C). In contrast, in LKB1-deficient hepatocytes, metformin failed to induce detectable AMPK and ACC phosphorylation, although AMPK and ACC proteins were expressed normally (Figure 7C).

We next explored the capacity of LKB1-deficient and control hepatocytes to produce glucose in response to metformin treatment. The absence of LKB1 greatly increased basal glucose production to levels similar to those observed with $\mathrm{Bt}_{2}$-cAMP stimulation in control hepatocytes (Figure $8 \mathrm{~A}$ ). $\mathrm{Bt}_{2}$-cAMP stimulation increased glucose production even further in LKB1-deficient hepatocytes to levels higher than those in $\mathrm{Bt}_{2}$-cAMP-stimulated control hepatocytes (Figure 8A). Treatment with metformin inhibited $\mathrm{Bt}_{2}$-cAMP-stimulated glucose production in a dose-dependent manner in both LKB1-deficient and control hepatocytes (Figure 8A).

To study the influence of LKB1 deficiency on gluconeogenesis, we examined the expression of key gluconeogenic genes in response to metformin. Under basal conditions, expression of the genes 
A
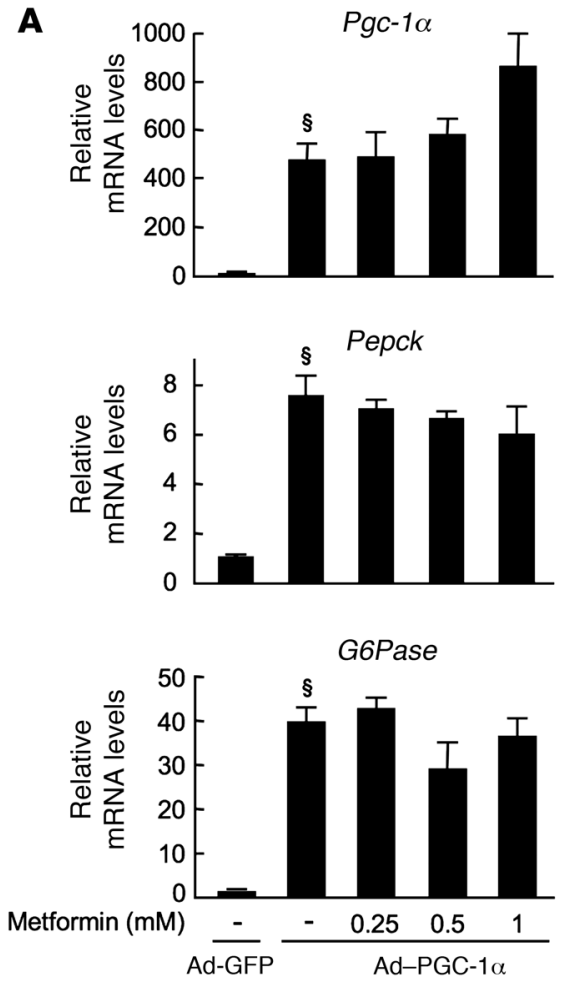

B

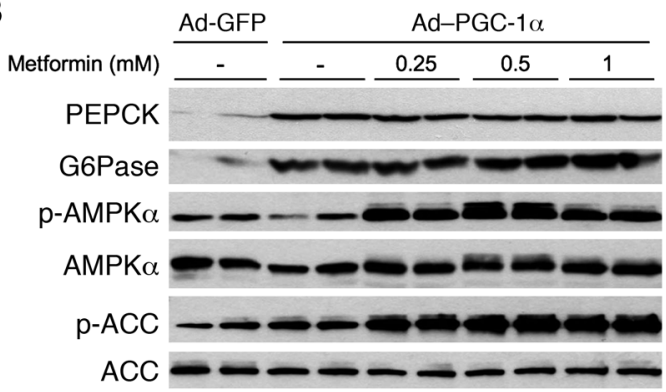

C

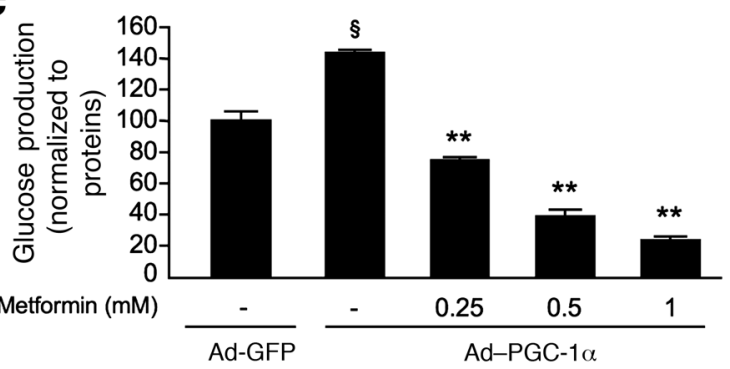

D

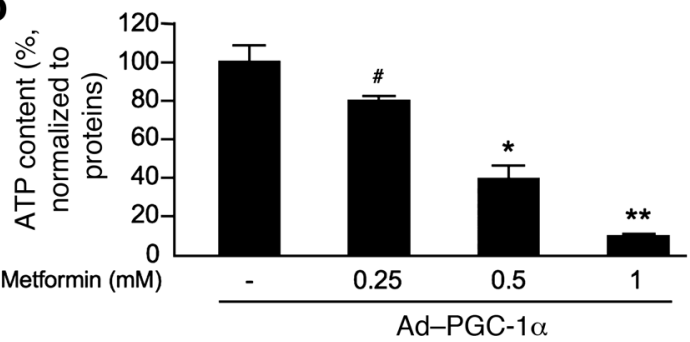

Figure 9

Forced expression of gluconeogenic genes does not prevent the metformin-induced inhibition of hepatic glucose production. After attachment, WT primary hepatocytes were infected with 25 PFU/cell of Ad-GFP or Ad-PGC-1 $\alpha$ adenovirus and cultured for 16 hours in M199 medium containing $100 \mathrm{nM}$ dex. Hepatocytes were then incubated in glucose-free DMEM containing lactate/pyruvate $(10: 1 \mathrm{mM}) \mathrm{and} 100 \mathrm{nM}$ dex alone or with $0.25,0.5$, or $1 \mathrm{mM}$ metformin. After 8 hours, medium was collected for glucose measurement and cells were harvested for Western blot and gluconeogenic gene expression analyses and ATP content determination. (A) Relative mRNA levels of Pgc-1 $\alpha, P e p c k$, and G6Pase expressed as fold activation relative to levels in Ad-GFP-infected hepatocytes. Results represent 3 independent experiments. $\$ P<0.001$ compared with Ad-GFP-infected hepatocytes. (B) Immunoblots were performed against PEPCK, G6Pase, phospho-AMPK $\alpha$ (Thr172), AMPK $\alpha$, phospho-ACC (Ser79), and ACC. Blots represent of 3 independent experiments. (C) Glucose production and (D) ATP intracellular content were normalized to protein content and expressed as a percentage of that produced by Ad-GFP- or Ad-PGC-1 $\alpha$-infected hepatocytes incubated in the absence of metformin. Results represent 5 independent experiments. ${ }^{~} P<0.01$ compared with Ad-GFP-infected hepatocytes; ${ }^{*} P<0.05$, ${ }^{\star} P<0.01$, ${ }^{\star \star} P<0.001$ compared with Ad-PGC-1 $\alpha$-infected hepatocytes incubated in the absence of metformin. Data are mean \pm SEM.

encoding PGC-1 $\alpha$, G6Pase, and PEPCK was markedly increased in LKB1-deficient compared with control hepatocytes (Figure 8B). $\mathrm{Bt}_{2}$-cAMP stimulation further increased expression of the gene encoding PGC-1 $\alpha$, but not G6Pase and PEPCK, in LKB1-deficient hepatocytes (Figure 8B). Despite enhanced Pgc-1 $\alpha$ gene expression after metformin treatment in LKB1-deficient hepatocytes, expression of the G6Pase gene was inhibited, whereas Pepck mRNA levels remained unaffected (Figure $8 \mathrm{~B}$ ). At the protein level, amounts of G6Pase and PEPCK were considerably increased under basal conditions and were not altered by metformin treatment (Figure 7C). We next examined whether CRTC2 phosphorylation was affected in LKB1-deficient hepatocytes with or without metformin. In contrast to the results obtained with AMPK-deficient hepatocytes, CRTC2 appeared to be predominantly dephosphorylated in basal conditions in LKB1-deficient hepatocytes, consistent with a faster electrophoretic mobility of CRTC2 from LKB1-deficient hepatocytes than that from control hepatocytes (Figure 7C). Met- formin failed to induce CRTC2 phosphorylation in the absence of LKB1 (Figure 7C). The CRTC2 protein content was lower in LKB1deficient hepatocytes than in the control hepatocytes. These data show that deletion of LKB1 enhanced gluconeogenesis in basal conditions, whereas LKB1 was dispensable for the inhibitory effect of metformin on gluconeogenesis.

Control of hepatic gluconeogenesis by AICAR in the absence of LKB1. Isolated hepatocytes from liver-specific $L k b 1$-KO mice were stimulated with $\mathrm{Bt}_{2}$-cAMP and incubated with increasing concentrations of AICAR to examine the role of LKB1 in AICAR-induced inhibition of hepatic gluconeogenesis. Stimulation of glucose production was markedly attenuated by AICAR in a dose-dependent manner in both LKB1-deficient and control hepatocytes (Supplemental Figure 6A). We then examined whether LKB1 was involved in the AICAR activation of AMPK by measuring levels of phosphorylated AMPK and ACC. As shown in Supplemental Figure 6B, AICAR-induced phosphorylation of both AMPK and ACC was abolished in the absence of LKB1. Similarly, 
CRTC2 phosphorylation was absent in LKB1-deficient hepatocytes treated with AICAR (Supplemental Figure 6B). In LKB1-deficient hepatocytes, Pgc-1 $\alpha$, G6Pase, and Pepck gene expression was decreased by AICAR in a pattern similar to that observed in control hepatocytes (Supplemental Figure 6C). These results indicate that AICAR action on hepatic gluconeogenesis is LKB1 independent.

Metformin suppresses hepatic glucose production without affecting gluconeogenic gene expression. It has been suggested that metformin acts upstream of PGC-1 $\alpha$ to inhibit hepatic glucose production by activating $A M P K$ via $\operatorname{LKB} 1(10,22)$. We reasoned that the overexpression of PGC- $1 \alpha$ could bypass the inhibitory effect of metformin on hepatic glucose production. To test this hypothesis, we infected wild-type hepatocytes with an adenovirus encoding PGC-1 $\alpha$ and measured the effects of metformin on gluconeogenesis. As expected, PGC-1 $\alpha$ overexpression led to upregulation of Pepck and G6Pase gene expression (Figure 9A). In addition, overexpression of PGC-1 $\alpha$ resulted in an increase in PEPCK and G6Pase protein levels (Figure 9B) and glucose production (Figure $9 \mathrm{C}$ ). Incubation with increasing concentrations of metformin increased AMPK and ACC phosphorylation in a dosedependent manner but was unable to repress gluconeogenic gene expression (Figure 9A). Importantly, under these conditions, metformin still inhibited glucose production, indicating that metformin suppresses gluconeogenesis independently of PGC- $1 \alpha$ through a transcription-independent process. Again, inhibition of glucose production by metformin was associated with a significant decrease in ATP content in a dose-dependent manner (Figure 9D).

\section{Discussion}

Increased hepatic glucose production is a major cause of hyperglycemia in type 2 diabetes. Metformin has been used for decades to improve glycemic control in diabetic patients and is thought to decrease blood glucose levels by reducing hepatic glucose output $(4,5)$. While metformin is currently the drug of choice for the treatment of type 2 diabetes, the precise mechanisms of its molecular action are not well understood. The most widely accepted mechanism of metformin action is the inhibition of transcription of key gluconeogenic genes in the liver $(10,22)$. It has been proposed that metformin stimulates CRTC2 phosphorylation in response to metabolic signals such as energy stress through the LKB1-AMPK/SIK1 pathways, which promotes binding to $14-3-3$ proteins, thereby sequestering CRTC2 from the nucleus to the cytoplasm $(10,18)$. If this is the case, genetic deletion of AMPK or LKB1 would be expected to lead to altered CRTC2 regulation. Under basal condition, the levels of CRTC2 phosphorylation as well as the levels of PEPCK and G6Pase were similar in AMPK $\alpha 1 \alpha 2$-null and control hepatocytes. In contrast, in LKB1-deficient hepatocytes, CRTC2 was markedly dephosphorylated, and this was associated with a dramatic increase in the expression of gluconeogenic genes without cAMP stimulation. Lack of CRTC2 phosphorylation by the LKB1-regulated protein kinases SIK1 (20) and MARK2 (21) at distinct regulatory sites could explain the phosphorylation-dependent mobility shift of CRTC2 and the activation of gluconeogenic genes. Thus, our data show LKB1-dependent, but AMPK-independent, phosphorylation and regulation of basal CRTC2 activity in primary hepatocytes. Normal fasted glycemia and glucose tolerance in AMPK $\alpha 1 \alpha 2_{\mathrm{LS}^{-/-}}$mice support these data (Figure 2, B, F, and $G$ ). This is quite different from results from mice with LKB1deficient livers, which exhibit hyperglycemia and glucose intolerance (10), indicating the critical role of LKB1-dependent pathway, but not AMPK in the liver in the control of gluconeogenesis.
We demonstrated that the metformin-induced phosphorylation of CRTC2 was abolished in hepatocytes lacking AMPK and LKB1 (Figure 1B and Figure 7C), indicating that upon stimulation, LKB1 and AMPK pathways could both regulate CRTC2 activity. Unexpectedly, in the absence of CRTC2 phosphorylation, hepatic glucose production was still repressed by metformin in both AMPKand LKB1-deficient hepatocytes. In addition, the repression of G6Pase gene expression in response to metformin treatment was preserved in hepatocytes deleted for AMPK or LKB1 (Figure 1C and Figure $8 \mathrm{~B}$ ). These results suggest that phosphorylation of CRTC2 by AMPK is not required in order to cause the inhibition of G6Pase gene expression by metformin. Thus, alternative pathways are likely to be involved in the control of gluconeogenesis by metformin. In support of this hypothesis, recent data indicated that metformin inhibits gluconeogenesis independent of CRTC2 phosphorylation at Ser171. The importance of CBP phosphorylation in the therapeutic effects of metformin was highlighted (22). The action of metformin was shown to be mediated through activation of $\mathrm{aPKC} / \lambda$, which phosphorylates CBP at Ser436, and disrupts the transcriptionally active CREB-CBP-CRTC2 complex, leading to the repression of gluconeogenic gene expression (22). It has been suggested that the CREB-CBP-CRTC2 complex functions as a regulator of gluconeogenesis, and it is therefore reasonable to assume that multiple signaling pathways regulate the cellular activity of this complex.

Our results indicate that metformin may inhibit glucose production independent of transcriptional repression of gluconeogenic genes. Indeed, significant reduction in glucose production occurred at concentrations of metformin of $0.25 \mathrm{mM}$ in control, AMPK-, and LKB1-deficient hepatocytes, while the expression of Pepck and G6Pase genes was not affected (Figure 1, A and C, and Figure 8, A and B). In particular, metformin had no significant effect on Pepck gene expression in control, AMPK-, and LKB1deficient hepatocytes (Figure 1C and Figure 8B). Similarly, a lack of regulation of Pepck gene expression was recently reported following 1 and 5 days of metformin treatment in diabetic $o b / o b$ mice despite a significant reduction in blood glucose levels (32). However, metformin decreased G6Pase gene expression considerably in control, AMPK-, and LKB1-deficient hepatocytes, but this decrease was not associated with a significant change in G6Pase protein levels (Figure 1B and Figure 7C). This suggests that repression of G6Pase gene expression alone could not explain the inhibitory effect of metformin on glucose production. Moreover, we demonstrated a substantial reduction in glucose production with metformin treatment, despite forced expression of key gluconeogenic genes through PGC- $1 \alpha$ overexpression (Figure 9C). These data conflict with the recently reported mechanism of metformin action, which involves the disassembly of the CREBCBP-CRTC2 transcription complex at Pgc-1 $\alpha$ and Pepck promoters (22). Thus, we consider that metformin action is related to a negative action on gluconeogenic flux rather than direct inhibition of gluconeogenic gene expression. The suppression of hepatic glucose production by metformin in insulin-resistant high-fat diet-fed rats is dependent on an inhibition of the substrate flux through G6Pase, and not on a decrease in the amount of enzyme (42), which supports our conclusion.

Metformin inhibits complex I of the respiratory chain in intact cells $(37,38)$ but does not affect the oxidative phosphorylation machinery downstream of complex I (37). The metformininduced respiratory inhibition does not involve the formation 
of $\mathrm{NO}$, ceramide, and oxygen radicals, or $\mathrm{Ca}^{2+}$ homeostasis (37). Although the exact pathway involved in the metformin inhibition of complex I is not well understood, it is thought that inhibition of gluconeogenesis by metformin, in intact animals and in isolated hepatocytes, results from a disruption of energy metabolism (37, 38). Supporting this hypothesis, previous work demonstrated a marked reduction of ATP/ADP ratio in both cytosolic and mitochondrial compartments in rat hepatocytes exposed to metformin $(37,38,43)$. Moreover, ATP levels were significantly decreased after treatment of primary hepatocytes with $0.25 \mathrm{mM}$ metformin (Figures 5 and 6), and were substantially equivalent to those present in the liver of metformin-treated mice (26). Of note, at higher concentrations, metformin has adverse effect on hepatocytes, since it significantly decreases total adenine nucleotide pools (Figure 5C). This adverse effect is reminiscent of an occasional side effect of metformin: the production of lactic acidosis by increased glycolytic activity in diabetic patients, which occurs if inhibition of the respiratory chain by the drug treatment becomes excessive. Indeed, severe lactic acidosis was the reason for withdrawal of the more potent phenformin as a clinical antidiabetic. Metformin-induced changes in the hepatic energy charge also occur in vivo, as demonstrated by the substantial increase in AMP/ATP ratio in the livers of mice after administration of $20 \mathrm{mg} / \mathrm{kg}$ metformin, a dose typically used clinically (Figure 5G). In addition, another study reported a decrease in ATP/ADP ratio in the liver of rats treated with metformin (38). This strongly suggests that metformin indirectly stimulates AMPK through a decrease in ATP levels. The fall in ATP was more pronounced in AMPK $\alpha 1 \alpha 2$-null than in control hepatocytes, resulting in the marked inhibition of glucose production (Figure 1A, Figure 6, and Supplemental Figure 2). This could be related to the decrease in mitochondrial content observed in AMPK-deficient hepatocytes (29), suggesting an apparent alteration of the mitochondrial biogenesis program in the absence of AMPK. This notion was further supported by the observation of AMPK-dependent regulation of $P g c-1 \alpha$ gene expression by metformin (Figure 1, A and C). This may be linked to the critical role of AMPK in cellular adaptation during energy deficiency of various origins, but is probably the result of impaired mitochondrial biogenesis due to a lack of increase in PGC-1 $\alpha$ expression and activity $(44,45)$. Therefore, we suggest that the fall in ATP concentration is sufficient to explain the inhibition of glucose production by metformin, without alteration in the expression of key enzymes involved in gluconeogenesis. In addition, as ATP and AMP vary reciprocally in the cell due to the reaction catalyzed by adenylate kinase $(2 \mathrm{ADP} \leftrightarrow \mathrm{ATP}+\mathrm{AMP})$, which is maintained close to the equilibrium, AMP tends to rise whenever ATP falls (Figure 5, A and F). AMP is a potent allosteric inhibitor of fructose 1,6-bisphosphatase, a key enzyme in gluconeogenesis (32). Therefore, metformininduced inhibition of glucose output could be attributable to both ATP depletion and a concomitant rise in AMP intracellular levels.

The rate of hepatic glucose production is closely linked to hepatic energy metabolism, and disruption of the principal source of energy in the hepatocytes (mitochondrial oxidative phosphorylation) would have a profound effect on the flux through gluconeogenesis (Figure 5E and refs. 46, 47). This is reminiscent of the observed defects in hepatic gluconeogenesis in both Pgc-1 $\alpha$ - and Ppara-KO mice $(48,49)$. For instance, impaired hepatic energy production in the absence of PGC-1 $\alpha$ inhibited the energetically costly process of gluconeogenesis and did not correlate with altered expression of key enzymes involved in gluconeogenesis.
We clearly showed that AICAR-induced inhibition of hepatic glucose production and gluconeogenic gene expression was independent of AMPK activity (Figure 3A). Similar results have been reported in studies on hepatocytes derived from the regulatory subunit AMPK $\beta 1$-deleted animals (35). Different mechanisms have been proposed to explain the AMPK-independent effect of AICAR on hepatic glucose production. AICAR does not activate AMPK directly and has to be converted to the AMP analog ZMP. Intracellular accumulation of ZMP has been reported to directly modulate fructose 1,6-bisphosphatase and gluconeogenic flux (50). Furthermore, there is evidence that AICAR inhibits mitochondrial oxidative phosphorylation and thus causes a decrease in ATP content in primary hepatocytes $(29,40,51)$. Unlike AICAR, A-769662 is a direct and potent activator of AMPK $(32,33)$ that does not cause significant alterations in AMP/ATP ratio in cells or inhibit fructose 1,6-bisphosphatase in cell-free assays $(32,36)$. It was therefore surprising that A-769662 did not inhibit glucose production and gluconeogenic gene expression in hepatocytes (Figure 4, B and C). Our results conflict with a previous report showing a blood glucose-lowering effect of A-769662 in obese $o b / o b$ mice (32). However, since A-769662 treatment was shown to dramatically reduce hepatic steatosis in these mice (32), a reduction in hepatic lipid content may have enhanced insulin sensitivity and thus insulin action in the liver, improving the control of hepatic glucose production. This hypothesis may also offer a potential explanation for why in liver-specific Lkb1-KO mice fed a high-fat diet, treatment with metformin failed to induce lowering of blood glucose levels (10). Indeed, in this study, it is difficult to establish whether metformin acts directly on the regulation of gluconeogenesis or indirectly by improving liver steatosis imposed by high-fat diet (thereby reducing lipotoxicity and insulin resistance known to enhance hepatic glucose production). Accordingly, evidence that metformin improved fatty liver disease, reversing hepatic steatosis in $o b / o b$ mice $(32,52)$ but also in rodents fed a high-fat diet (53), has been reported. Metformin-induced reduction in hepatic lipid content is consistent with an increase in fatty acid oxidation presumably acting through hepatic AMPK activation $(32,54)$. Thus, impaired metformin-induced AMPK phosphorylation in the absence of LKB1 (10) blunts the improvements in hepatic lipid content and insulin sensitivity in LKB1-deficient mice, impeding the expected normalization of blood glucose levels. Importantly, these observations call into question the action of AMPK in direct control of hepatic gluconeogenesis. In response to prolonged fasting, the liver is the main source of energy substrates for extrahepatic organs to maintain the whole-body energy balance. It produces ketone bodies and glucose by hepatic $\beta$-oxidation and gluconeogenesis, respectively. It has been demonstrated that during the fed-starved transition, AMPK is activated in the liver and contributes to the switch between lipogenesis and $\beta$-oxidation as a result of its energy sensor function (55). In contrast, AMPKmediated inhibition of hepatic glucose production during fasting may appear paradoxical when blood glucose levels should be maintained in a narrow range. Indeed, it is intuitively appealing to hypothesize that maintenance of glycemia must take precedence over hepatic energy balance during prolonged fasting. Thus, further study is required to clarify the direct role of AMPK in the liver in regulating hepatic glucose production.

In conclusion, our results provide a molecular insight into the regulation of hepatic glucose production by metformin. Using genetic mouse models, we demonstrate the crucial role for LKB1 but not 
AMPK in the control of basal hepatic glucose production. We also provide evidence that metformin inhibits gluconeogenesis through LKB1- and AMPK-independent pathways. The metformin-induced inhibition of glucose production occurs through regulation of the gluconeogenesis flux rather than direct inhibition of gluconeogenic gene expression. Moreover, we show that the control of hepatic glucose production by metformin is linked to the inhibition of gluconeogenesis in response to a decrease of the hepatic energy state. Our data and recent findings $(56,57)$ suggest that the development of small molecules targeting mitochondrial function in the liver, thus causing a moderate variation in hepatic energy charge, is an attractive therapeutic strategy for the treatment of type 2 diabetes.

\section{Methods}

Animals. Mice were maintained under a 12-hour light/12-hour dark cycle with free access to water and standard mouse diet (in terms of energy: $65 \%$ carbohydrate, $11 \%$ fat, $24 \%$ protein). C57BL $/ 6 \mathrm{~J}$ mice were obtained from Charles River Laboratories France. Mice lacking both AMPK $\alpha 1$ and AMPK $\alpha 2$ catalytic subunits were generated by crossing AMPK $\alpha 1^{-/-}$and liver-specific AMPKo2 $2^{-/-}$mice as previously described (29). For hepatic Lkb1 gene deletion, Lkb1-floxed mice (41) were anesthetized with isoflurane and were injected with adenovirus Cre-GFP or adenovirus GFP into the penis vein $\left(5 \times 10^{8} \mathrm{PFU} /\right.$ mouse $)$. Littermates from the same breeding pair were used in these experiments. Hepatocytes were isolated and cultured 2 weeks after adenovirus injection. All procedures were performed in accordance with the principles and guidelines established in the European Convention for the Protection of Vertebrate Animals Used for Experimental and Other Scientific Purposes (Council of Europe, ETS no. 123, 1991). Animal studies described herein were reviewed and approved (agreement no. 75-886) by the Directeur Départemental des Services Vétérinaires of the Préfecture de Police de Paris.

Glucose, insulin, metformin, and pyruvate tolerance tests. Oral glucose tolerance tests were performed on age-matched male mice fasted for 16 hours as previously described (58). Blood glucose levels were determined at 0, 20, 40, and 60 minutes after oral administration of glucose ( $3 \mathrm{~g} / \mathrm{kg}$ body weight) using a glucometer (Glucotrend II; Roche Diagnostics). For metformin tolerance tests, mice fasted for 16 hours were given an oral gavage dose of 50,150 , or $300 \mathrm{mg} / \mathrm{kg}$ metformin and then after 30 minutes challenged with an oral administration of glucose $(3 \mathrm{~g} / \mathrm{kg}$ body weight). For insulin tolerance tests, animals fasted for 5 hours were injected intraperitoneally with insulin $(0.25 \mathrm{U} / \mathrm{kg}$ body weight; Actrapid, Novo Nordisk), and blood glucose levels were measured at $0,15,30,45$, and 60 minutes after injection. For pyruvate tolerance tests, animals fasted for 16 hours were injected intraperitoneally with pyruvate ( $2 \mathrm{~g} / \mathrm{kg}$ body weight) dissolved in saline, and blood glucose levels were measured as above.

Plasma glucose and insulin levels. Blood glucose and insulin levels of mice fed ad libitum or fasted were analyzed. We collected blood from mice in the fed state, at 9:00 p.m. by tail bleeding (58). For fasting experiments, food was removed at 5:00 p.m., and the mice were kept in a clean new cage for 5 hours before blood collection (58). Serum insulin concentrations were assessed using a rat or mouse insulin ultrasensitive enzyme-linked immunosorbent assay kit with mouse insulin as standard (Crystal Chem).

Adenoviruses. Adenovirus expressing mouse PGC- $1 \alpha$ was generated as described by He et al. (59). Briefly, the PGC- $1 \alpha$ cDNA was amplified by PCR from mouse liver cDNA then subcloned into the shuttle vector pAdTrackCMV. The resultant plasmid was linearized by the restriction endonuclease PmeI and used to transform the Escherichia coli strain BJ5183-AD1 (Stratagene) containing the supercoiled adenoviral vector pAd-Easy1. Recombinants were selected by kanamycin resistance and screened by restriction endonuclease digestion. The recombinant adenoviral construct was digested with PacI and used to transfect the packaging cell line Ad-293 (Stratagene) with Lipofectamine 2000 (Invitrogen) to produce virus. Ad-Cre-GFP was a gift from S. Colnot (INSERM U1016, Paris, France) (60). Adenovirus expressing GFP was used as a control. Adenoviruses were propagated in Ad-293 cells and purified by cesium chloride density centrifugation as previously described (61).

Primary mouse hepatocytes. Primary hepatocytes were isolated from fed adult mice by a modified version of the collagenase method (62). The cells were plated in M199 medium supplemented with $100 \mathrm{U} / \mathrm{ml}$ penicillin, $100 \mu \mathrm{g} / \mathrm{ml}$ streptomycin, $0.1 \%$ bovine serum albumin, $2 \%$ Ultroser G (Biosepra), $500 \mathrm{nM}$ dexamethasone (dex; Sigma-Aldrich), $100 \mathrm{nM}$ triiodothyronine (SigmaAldrich), $10 \mathrm{nM}$ insulin (Actrapid, Novo Nordisk), at a density of $2.5 \times 10^{5}$ cells/well on 6-well plates or $2.5 \times 10^{6} \mathrm{cells} / 100$-mm-diameter cell culture plate. After attachment (3-4 hours), hepatocytes were maintained in M199 medium with antibiotics and $100 \mathrm{nM}$ dex for 16 hours, followed by drug treatment as described below. For experiments with adenovirus, hepatocytes were infected with $25 \mathrm{PFU} /$ cell of Ad-GFP or Ad-PGC-1 $\alpha$ after the attachment period in M199 medium containing antibiotics and $100 \mathrm{nM}$ dex for 16 hours.

Hepatocyte glucose production. Primary mouse hepatocytes on 6-well plates $\left(2.5 \times 10^{5}\right.$ cells/well $)$ were maintained in M199 medium containing antibiotics and $100 \mathrm{nM}$ dex for 16 hours prior to the measurement of glucose production. Hepatocytes were washed once with PBS, and glucose production was determined after a 4-, 8-, or 12-hour incubation period in glucose-free DMEM containing lactate/pyruvate $(10: 1 \mathrm{mM})$ or $10 \mathrm{mM}$ dihydroxyacetone and $100 \mathrm{nM}$ dex alone or with $100 \mu \mathrm{M} \mathrm{Bt}_{2}$-cAMP (Sigma-Aldrich) and with various doses of metformin (Sigma-Aldrich), AICAR (Toronto Research Chemicals), or A-769622 as indicated in the figure legends. A-769662 compound was synthesized as described previously (33). At the end of the incubation period, 1 or $1.5 \mathrm{ml}$ of medium was collected and mixed with $0.2 \mathrm{ml}$ $\mathrm{HClO}_{4}(40 \% \mathrm{v} / \mathrm{v})$. After neutralization, the amount of glucose released into the medium was determined by evaluating the production of NADPH from NADP in the presence of hexokinase and glucose-6-phosphate dehydrogenase (Roche) and normalized to the total protein content per well.

Measurement of adenine nucleotide levels. Adenine nucleotide concentrations were determined in cell extracts prepared from cultured hepatocytes or from liver samples by an enzymatic method (63). Primary hepatocytes were treated as described in the figure legends, the culture medium was removed, and cells on 100 -mm-diameter cell culture plates $\left(2.5 \times 10^{6}\right.$ cells/plate $)$ were scraped into $200 \mu \mathrm{l}$ of $6 \%(\mathrm{v} / \mathrm{v})$ ice-cold $\mathrm{HClO}_{4}$ in less than 5 seconds. For liver, 10-week-old C57BL/6J male mice were treated by oral intubation with 20 or $50 \mathrm{mg} / \mathrm{kg}$ metformin in water $(200 \mu \mathrm{l})$ or just water directly into the stomach for 5 consecutive days. On the fifth day, 24-hour-fasted mice were sacrificed by cervical dislocation 1 hour after metformin administration, and liver was extracted and frozen in liquid nitrogen in less than 25 seconds. Two hundred milligrams of liver was homogenized into $1 \mathrm{ml}$ of $6 \%(\mathrm{v} / \mathrm{v})$ ice-cold $\mathrm{HClO}_{4}$. Cell extracts were centrifuged at $10,000 \mathrm{~g}$ for 10 minutes at $4^{\circ} \mathrm{C}$. The acid supernatant was neutralized and used for spectrophotometric determination of adenine nucleotides. Standard curves for ATP, $\mathrm{ADP}$, and AMP were constructed with $25,50,75,100,125$, and $150 \mu \mathrm{M}$ of each nucleotide. Adenine nucleotide levels were expressed in $\mathrm{nmol} / 10^{6}$ hepatocytes, $\mu \mathrm{mol} / \mathrm{g}$ liver weight, or percentage of control normalized to protein content. Energy charge was calculated by the following equation: $[\mathrm{ATP}+\mathrm{ADP} / 2] /[\mathrm{ATP}+\mathrm{ADP}+\mathrm{AMP}]$, where AMP, ADP, and ATP are the respective tissue concentrations (64).

Quantitative PCR analysis. Total RNA from primary hepatocytes and mouse liver tissue was extracted using Trizol (Invitrogen), and single-strand cDNA was synthesized from $5 \mu \mathrm{g}$ of total RNA with random hexamer primers (Applied Biosystems) and Superscript II (Invitrogen). Real-time RT-PCRs were carried out with Lithos qPCR MasterMix (Eurogenetec) in a final volume of $20 \mu \mathrm{l}$ containing $250 \mathrm{ng}$ of reverse-transcribed total RNA, $500 \mathrm{nM}$ of primers, $10 \mu \mathrm{l}$ of $2 \times$ PCR mix, and $0.5 \mu \mathrm{l}$ of Sybr Green. The reactions 
were carried out in capillaries in a LightCycler instrument (Roche) with 40 cycles. We determined the relative amounts of the mRNAs studied by means of the second-derivative maximum method, with LightCycler analysis software version 3.5 and 18S RNA as the invariant control for all studies. The sense and antisense PCR primers used, respectively, were as follows: for $P g c-1 \alpha, 5^{\prime}$-ATACCGCAAAGAGCACGAGAAG-3', 5'-CTCAAGAGCAGCGAAAGCGTCACAG-3'; for Pepck, 5'-GTGCTGGAGTGGATGTTCGG-3', 5' -CTGGCTGATTCTCTGTTTCAGG-3'; for G6Pase, 5' -ACTGTGGGCATCAATCTCCTC-3' ' 5'-CGGGACAGACAGACGTTCAGC-3'; for 18S, 5'GTAACCCGTTGAACCCCATT-3' , 5'-CCATCCAATCGGTAGTAGCG-3' .

Western blot analysis. After an 8-hour incubation period, cultured hepatocytes were lysed in ice-cold lysis buffer containing $50 \mathrm{mM}$ Tris, pH 7.4, 1\% Triton X-100, $150 \mathrm{mM} \mathrm{NaCl}, 10 \%$ glycerol, $50 \mathrm{mM} \mathrm{NaF}, 5 \mathrm{mM}$ sodium pyrophosphate, $1 \mathrm{mM}$ $\mathrm{Na}_{3} \mathrm{VO}_{4}, 25 \mathrm{mM}$ sodium- $\beta$-glycerophosphate, $1 \mathrm{mM}$ DTT, $0.5 \mathrm{mM}$ PMSF, and protease inhibitors (Complete Protease Inhibitor Cocktail; Roche). Hepatocyte lysates were sonicated on ice for 20 seconds. The liver was homogenized in icecold lysis buffer using a ball-bearing homogenizer (Retsch). The homogenate was centrifuged for 10 minutes at $10,000 \mathrm{~g}$ at $4^{\circ} \mathrm{C}$, and the supernatants were removed for determination of total protein content. Fifty micrograms of protein from the supernatant was separated on $7.5 \%$ or $10 \%$ SDS-PAGE gels and transferred to nitrocellulose membranes. The membranes were blocked for 30 minutes at $37^{\circ} \mathrm{C}$ with Tris-buffered saline supplemented with $0.05 \%$ NP40 and 5\% nonfat dry milk. Immunoblotting was performed following standard procedures, and the signals were detected by chemiluminescence reagents (Thermo). Primary antibodies were directed against: total AMPK $\alpha$, AMPK $\alpha$ phosphorylated at Thr172, total ACC, ACC phosphorylated at Ser79, phospho-PKA substrate (all from Cell Signaling Technology), total LKB1 (Abcam), total CRTC2 (Calbiochem), PEPCK (a gift from S. Colnot), $\beta$-actin (clone AC.74; Sigma-Aldrich). Antibodies against total G6Pase were described previously (65). The LKB1 antibody used for immunoprecipitation was raised in sheep against the $\mathrm{NH}_{2}$-terminal peptide TFIHRIDSTEVIYQPR of human LKB1, residues 24-39 (sheep number 611A).

LKB1 activity. Cultured hepatocytes were lysed in ice-cold lysis buffer containing 50 mM Tris-HCl pH 7.5, 1 mM EGTA, 1 mM EDTA, 1\% Triton X-100,
$1 \mathrm{mM}$ sodium orthovanadate, $50 \mathrm{mM}$ sodium fluoride, $5 \mathrm{mM}$ sodium pyrophosphate, $0.27 \mathrm{M}$ sucrose, $0.1 \% \beta$-mercaptoethanol, and protease inhibitors (Complete Protease Inhibitor Cocktail; Roche). LKB1 activity was measured as previously described using LKBtide (human NUAK2 $241-260$ with 3 additional Arg residues) as substrate (66).

Statistics. Results are expressed as mean \pm SEM. Comparisons between groups were made by unpaired 2-tailed Student's $t$ test. Differences were considered statistically significant if $P$ was less than 0.05 .

\section{Acknowledgments}

This work was supported by the European Commission integrated project (LSHM-CT-2004-005272/exgenesis), Association pour l'Etude des Diabètes et des Maladies Métaboliques (ALFEDIAM), Programme National de Recherche sur le Diabète (PNRD), Association de Recherche sur le Diabète (ARD), Institut Benjamin Delessert, Association pour la Recherche sur le Diabète, l'Insuffisance cérébrale et le Cancer (AREDIC), and Institut Appert. We thank Region Ile de France for contributing to the Cochin Institute animal care facility. K. Sakamoto was supported by Diabetes UK (07/0003529), Dundee and District Diabetes UK Volunteer Group, the UK Medical Research Council, and the Swedish Foundation for International Cooperation in Research and Higher Education. We also thank the antibody purification teams (Division of Signal Transduction Therapy [DSTT], University of Dundee) coordinated by Hilary McLauchlan and James Hastie.

Received for publication August 3, 2009, and accepted in revised form May 20, 2010.

Address correspondence to: Benoit Viollet or Marc Foretz, Institut Cochin, Département d'Endocrinologie Métabolisme et Cancer, 24, rue du Faubourg Saint Jacques, 75014 Paris, France. Phone: 33.1.44.41.24.01; Fax: 33.1.44.41.24.21; E-mail: benoit.viollet@ inserm.fr (B. Viollet); marc.foretz@inserm.fr (M. Foretz).
1. Rotella CM, Monami M, Mannucci E. Metformin beyond diabetes: new life for an old drug. Curr Diabetes Rev. 2006;2(3):307-315.

2. Crandall JP, et al. The prevention of type 2 diabetes. Nat Clin Pract Endocrinol Metab. 2008;4(7):382-393.

3. Klip A, Leiter LA. Cellular mechanism of action of metformin. Diabetes Care. 1990;13(6):696-704.

4. Bailey CJ, Turner RC. Metformin. N Engl J Med. 1996;334(9):574-579.

5. Kirpichnikov D, McFarlane SI, Sowers JR. Metformin: an update. Ann Intern Med. 2002;137(1):25-33.

6 . Hundal RS, et al. Mechanism by which metformin reduces glucose production in type 2 diabetes. Diabetes. 2000;49(12):2063-2069.

7. Shu Y, et al. Effect of genetic variation in the organic cation transporter 1 (OCT1) on metformin action. J Clin Invest. 2007;117(5):1422-1431.

8. Zhou G, et al. Role of AMP-activated protein kinase in mechanism of metformin action. JClin Invest. 2001; 108(8):1167-1174.

9. Musi N, et al. Metformin increases AMP-activated protein kinase activity in skeletal muscle of subjects with type 2 diabetes. Diabetes. 2002;51(7):2074-2081.

10. Shaw RJ, et al. The kinase LKB1 mediates glucose homeostasis in liver and therapeutic effects of metformin. Science. 2005;310(5754):1642-1646.

11. Hardie DG. AMP-activated protein kinase as a drug target. Annu Rev Pharmacol Toxicol. 2007;47:185-210.

12. Viollet B, et al. AMP-activated protein kinase in the regulation of hepatic energy metabolism: from physiology to therapeutic perspectives. Acta Physiol (Oxf). 2009;196(1):81-98.

13. Hawley SA, et al. Complexes between the LKB1 tumor suppressor, STRAD alpha/beta and MO25 alpha/beta are upstream kinases in the AMP-activated protein kinase cascade. J Biol. 2003;2(4):28.

14. Shaw RJ, et al. The tumor suppressor LKB1 kinase directly activates AMP-activated kinase and regulates apoptosis in response to energy stress. Proc Natl Acad Sci U S A. 2004;101(10):3329-3335.

15. Woods A, et al. LKB1 is the upstream kinase in the AMP-activated protein kinase cascade. Curr Biol. 2003;13(22):2004-2008.

16. Hawley SA, et al. Calmodulin-dependent protein kinase kinase-beta is an alternative upstream kinase for AMP-activated protein kinase. Cell Metab. 2005; 2(1):9-19.

17. Woods A, et al. Ca2+/calmodulin-dependent protein kinase kinase-beta acts upstream of AMP-activated protein kinase in mammalian cells. Cell Metab. 2005; 2(1):21-33.

18. Koo SH, et al. The CREB coactivator TORC2 is a key regulator of fasting glucose metabolism. Nature. 2005;437(7062):1109-1111.

19. Dentin R, et al. Insulin modulates gluconeogenesis by inhibition of the coactivator TORC2. Nature. 2007; 449(7160):366-369.

20 . Horike N, et al. AMP-activated protein kinase activation increases phosphorylation of glycogen synthase kinase 3 beta and thereby reduces cAMP-responsive element transcriptional activity and phosphoenolpyruvate carboxykinase $\mathrm{C}$ gene expression in the liver. J Biol Chem. 2008;283(49):33902-33910.

21. Jansson D, Ng AC, Fu A, Depatie C, Al Azzabi M, Screaton RA. Glucose controls CREB activity in islet cells via regulated phosphorylation of TORC2. Proc
Natl Acad Sci U S A. 2008;105(29):10161-10166.

22. He L, et al. Metformin and insulin suppress hepatic gluconeogenesis through phosphorylation of CREB binding protein. Cell. 2009;137(4):635-646.

23. Lizcano JM, et al. LKB1 is a master kinase that activates 13 kinases of the AMPK subfamily, including MARK/PAR-1. EMBO J. 2004;23(4):833-843.

24. Sakamoto K, Goransson O, Hardie DG, Alessi DR. Activity of LKB1 and AMPK-related kinases in skeletal muscle: effects of contraction, phenformin, and AICAR. Am J Physiol Endocrinol Metab. 2004;287(2):E310-E317.

25. Sum CF, Webster JM, Johnson AB, Catalano C, Cooper BG, Taylor R. The effect of intravenous metformin on glucose metabolism during hyperglycaemia in type 2 diabetes. Diabet Med. 1992;9(1):61-65.

26. Wilcock C, Bailey CJ. Accumulation of metformin by tissues of the normal and diabetic mouse. Xenobiotica. 1994;24(1):49-57.

27. Wilcock C, Wyre ND, Bailey CJ. Subcellular distribution of metformin in rat liver.J Pharm Pharmacol. 1991;43(6):442-444.

28. Yin W, Mu J, Birnbaum MJ. Role of AMP-activated protein kinase in cyclic AMP-dependent lipolysis In 3T3-L1 adipocytes. J Biol Chem. 2003; 278(44):43074-43080.

29. Guigas B, et al. 5-Aminoimidazole-4-carboxamide1-\{beta\}-d-ribofuranoside and metformin inhibit hepatic glucose phosphorylation by an AMP-activated protein kinase-independent effect on glucokinase translocation. Diabetes. 2006;55(4):865-874.

30. Wilcock C, Bailey CJ. Sites of metformin-stimulated glucose metabolism. Biochem Pharmacol. 
1990;39(11):1831-1834.

31. Corton JM, Gillespie JG, Hawley SA, Hardie DG. 5-aminoimidazole-4-carboxamide ribonucleoside. A specific method for activating AMP-activated protein kinase in intact cells? Eur J Biochem. 1995; 229(2):558-565.

32. Cool B, et al. Identification and characterization of a small molecule AMPK activator that treats key components of type 2 diabetes and the metabolic syndrome. Cell Metab. 2006;3(6):403-416.

33. Goransson O, et al. Mechanism of action of A-769662, a valuable tool for activation of AMP-activated protein kinase. J Biol Chem. 2007;282(45):32549-32560.

34. Sanders MJ, Grondin PO, Hegarty BD, Snowden $\mathrm{MA}$, Carling D. Investigating the mechanism for AMP activation of the AMP-activated protein kinase cascade. Biochem J. 2007;403(1):139-148.

35. Scott JW, et al. Thienopyridone drugs are selective activators of AMP-activated protein kinase beta1-containing complexes. Chem Biol. 2008; 15(11):1220-1230.

36. Guigas B, et al. Beyond AICA riboside: in search of new specific AMP-activated protein kinase activators. IUBMB Life. 2009;61(1):18-26.

37. El-Mir MY, Nogueira V, Fontaine E, Averet N, Rigoulet M, Leverve X. Dimethylbiguanide inhibits cell respiration via an indirect effect targeted on the respiratory chain complex I. J Biol Chem. 2000; 275(1):223-228.

38. Owen MR, Doran E, Halestrap AP. Evidence that metformin exerts its anti-diabetic effects through inhibition of complex 1 of the mitochondrial respiratory chain. Biochem J. 2000;348(pt 3):607-614.

39. Bryla J, Harris EJ, Plumb JA. The stimulatory effect of glucagon and dibutyryl cyclic AMP on ureogenesis and gluconeogenesis in relation to the mitochondrial ATP content. FEBS Lett. 1977;80(2):443-448.

40. Guigas B, et al. AMP-activated protein kinase-independent inhibition of hepatic mitochondrial oxidative phosphorylation by AICA riboside. Biochem J. 2007;404(3):499-507.

41. Sakamoto K, et al. Deficiency of LKB1 in skeletal muscle prevents AMPK activation and glucose uptake during contraction. EMBO J. 2005;24(10):1810-1820.

42. Mithieux G, Guignot L, Bordet JC, Wiernsperger N Intrahepatic mechanisms underlying the effect of metformin in decreasing basal glucose production in rats fed a high-fat diet. Diabetes. 2002;51(1):139-143.

43. Argaud D, Roth H, Wiernsperger N, Leverve XM. Metformin decreases gluconeogenesis by enhancing the pyruvate kinase flux in isolated rat hepatocytes.
Eur J Biochem. 1993;213(3):1341-1348.

44. Zong $\mathrm{H}$, et al. AMP kinase is required for mitochondrial biogenesis in skeletal muscle in response to chronic energy deprivation. Proc Natl Acad Sci US A. 2002;99(25):15983-15987.

45. Baur JA, et al. Resveratrol improves health and survival of mice on a high-calorie diet. Nature. 2006;444(7117):337-342.

46. Owen MR, Halestrap AP. The mechanisms by which mild respiratory chain inhibitors inhibit hepatic gluconeogenesis. Biochim Biophys Acta. 1993; 1142(1-2):11-22.

47. Pryor HJ, Smyth JE, Quinlan PT, Halestrap AP. Evidence that the flux control coefficient of the respiratory chain is high during gluconeogenesis from lactate in hepatocytes from starved rats. Implications for the hormonal control of gluconeogenesis and action of hypoglycaemic agents. Biochem J. 1987;247(2):449-457.

48. Burgess SC, et al. Diminished hepatic gluconeogenesis via defects in tricarboxylic acid cycle flux in peroxisome proliferator-activated receptor gamma coactivator-1alpha (PGC-1alpha)-deficient mice. J Biol Chem. 2006;281(28):19000-19008.

49. Bandsma RH, et al. Hepatic de novo synthesis of glucose 6-phosphate is not affected in peroxisome proliferator-activated receptor alpha-deficient mice but is preferentially directed toward hepatic glycogen stores after a short term fast. J Biol Chem. 2004; 279(10):8930-8937.

50. Vincent MF, Marangos PJ, Gruber HE, Van den Berghe G. Inhibition by AICA riboside of gluconeogenesis in isolated rat hepatocytes. Diabetes. 1991;40(10):1259-1266.

51. Mukhtar $\mathrm{MH}$, et al. Inhibition of glucokinase translocation by AMP-activated protein kinase is associated with phosphorylation of both GKRP and 6-phosphofructo-2-kinase/fructose-2,6bisphosphatase. Am J Physiol Regul Integr Comp Physiol. 2008;294(3):R766-R774.

52. Lin HZ, Yang SQ, Chuckaree C, Kuhajda F, Ronnet G, Diehl AM. Metformin reverses fatty liver disease in obese, leptin-deficient mice. Nat Med. 2000; 6(9):998-1003.

53. Raso GM, et al. Comparative therapeutic effects of metformin and vitamin $\mathrm{E}$ in a model of non-alcoholic steatohepatitis in the young rat. EurJ Pharmacol. 2009;604(1-3):125-131.

54. Zang M, et al. AMP-activated protein kinase is required for the lipid-lowering effect of metformin in insulin-resistant human HepG2 cells.J Biol Chem.
2004;279(46):47898-47905.

55. Viollet B, et al. Activation of AMP-activated protein kinase in the liver: a new strategy for the management of metabolic hepatic disorders. J Physiol. 2006;574(pt 1):41-53.

56. Qiu BY, et al. High-throughput assay for modulators of mitochondrial membrane potential identifies a novel compound with beneficial effects on db/db mice. Diabetes. 2010;59(1):256-265.

57 . Turner N, et al. Berberine and its more biologically available derivative, dihydroberberine, inhibit mitochondrial respiratory complex I: a mechanism for the action of berberine to activate AMP-activated protein kinase and improve insulin action. Diabetes. 2008;57(5):1414-1418.

58. Viollet B, et al. The AMP-activated protein kinase alpha 2 catalytic subunit controls whole-body insulin sensitivity. J Clin Invest. 2003;111(1):91-98.

59. He TC, Zhou S, da Costa LT, Yu J, Kinzler KW, Vogelstein B. A simplified system for generating recombinant adenoviruses. Proc Natl Acad SciUS A. 1998;95(5):2509-2514.

60. Benhamouche $\mathrm{S}$, et al. Apc tumor suppressor gene is the "zonation-keeper" of mouse liver. Dev Cell. 2006;10(6):759-770.

61. Foretz M, Guichard C, Ferre P, Foufelle F. Sterol regulatory element binding protein-1c is a major mediator of insulin action on the hepatic expression of glucokinase and lipogenesis-related genes. Proc Natl Acad Sci U S A. 1999;96(22):12737-12742.

62 . Berry MN, Friend DS. High-yield preparation of isolated rat liver parenchymal cells: a biochemical and fine structural study. J Cell Biol. 1969;43(3):506-520.

63. Foretz M, Carling D, Guichard C, Ferre P, Foufelle F. AMP-activated protein kinase inhibits the glucoseactivated expression of fatty acid synthase gene in rat hepatocytes. J Biol Chem. 1998;273(24):14767-14771.

64. Atkinson DE, Walton GM. Adenosine triphosphate conservation in metabolic regulation. Rat liver citrate cleavage enzyme. J Biol Chem. 1967; 242(13):3239-3241.

65. Rajas F, Jourdan-Pineau H, Stefanutti A, Mrad EA, Iynedjian PB, Mithieux G. Immunocytochemical localization of glucose 6-phosphatase and cytosolic phosphoenolpyruvate carboxykinase in gluconeogenic tissues reveals unsuspected metabolic zonation. Histochem Cell Biol. 2007;127(5):555-565.

66. Sakamoto K, et al. Deficiency of LKB1 in heart prevents ischemia-mediated activation of AMPKalpha2 but not AMPKalpha1. Am J Physiol Endocrinol Metab. 2006;290(5):E780-E788. 